University of Warwick institutional repository: http://go.warwick.ac.uk/wrap This paper is made available online in accordance with publisher policies. Please scroll down to view the document itself. Please refer to the repository record for this item and our policy information available from the repository home page for further information.

To see the final version of this paper please visit the publisher's website. access to the published version may require a subscription.

Author(s): Ian Stewart, Martin Golubitsky and Marcus Pivato

Article Title: Symmetry Groupoids and Patterns of Synchrony in Coupled Cell Networks

Year of publication:2003

Link to published version:http://dx.doi.org/10.1137/S1111111103419896

Publisher statement: None 


\title{
Symmetry Groupoids and Patterns of Synchrony in Coupled Cell Networks*
}

\author{
Ian Stewart ${ }^{\dagger}$, Martin Golubitsky ${ }^{\ddagger}$, and Marcus Pivato $§$
}

Abstract. A coupled cell system is a network of dynamical systems, or "cells," coupled together. Such systems can be represented schematically by a directed graph whose nodes correspond to cells and whose edges represent couplings. A symmetry of a coupled cell system is a permutation of the cells that preserves all internal dynamics and all couplings. Symmetry can lead to patterns of synchronized cells, rotating waves, multirhythms, and synchronized chaos. We ask whether symmetry is the only mechanism that can create such states in a coupled cell system and show that it is not.

The key idea is to replace the symmetry group by the symmetry groupoid, which encodes information about the input sets of cells. (The input set of a cell consists of that cell and all cells connected to that cell.) The admissible vector fields for a given graph - the dynamical systems with the corresponding internal dynamics and couplings - are precisely those that are equivariant under the symmetry groupoid. A pattern of synchrony is "robust" if it arises for all admissible vector fields. The first main result shows that robust patterns of synchrony (invariance of "polydiagonal" subspaces under all admissible vector fields) are equivalent to the combinatorial condition that an equivalence relation on cells is "balanced." The second main result shows that admissible vector fields restricted to polydiagonal subspaces are themselves admissible vector fields for a new coupled cell network, the "quotient network." The existence of quotient networks has surprising implications for synchronous dynamics in coupled cell systems.

Key words. coupled systems, synchrony, groupoids, symmetry

AMS subject classifications. 34C14, 34C15, 20L05

DOI. $10.1137 / \mathrm{S} 1111111103419896$

1. Introduction. We use the term cell to indicate a system of ODEs. A coupled cell system is a set of cells with coupling, that is, a dynamical system whose variables correspond to cells, such that the output of certain cells affects the time-evolution of other cells. The salient feature of a coupled cell system is that the output from each cell is considered to be significant in its own right. A coupled cell system is not merely a system of ODEs but a system of ODEs equipped with canonical observables - the individual cells (see [8]). From a mathematical point of view these output signals can be compared, and this observation leads to a variety of notions of "synchrony." For surveys, see Boccaletti, Pecora, and Pelaez [2] and Wang [14].

In this paper we discuss the architecture of a coupled cell system: which cells influence which, which cells are "identical," and which couplings are "identical." We focus on how the

${ }^{*}$ Received by the editors February 19, 2003; accepted for publication (in revised form) by G. Kriegsmann August 29, 2003; published electronically December 22, 2003.

http://www.siam.org/journals/siads/2-4/41989.html

${ }^{\dagger}$ Mathematics Institute, University of Warwick, Coventry CV4 7AL, UK (ins@maths.warwick.ac.uk).

${ }^{\ddagger}$ Department of Mathematics, University of Houston, Houston, TX 77204-3476 (mg@uh.edu). The work of this author was supported in part by NSF grants DMS-0071735 and DMS-0244529 and ARP grant 003652-0032-2001.

${ }^{\S}$ Department of Mathematics, Trent University, Peterborough, ON, Canada K9L 1 Z6 (pivato@xaravve.trentu.ca). 
system architecture leads naturally to synchrony. To do this, we must define carefully when two cells or two couplings are "identical" or "equivalent." Indeed, the main point of this paper is to provide a general mathematical foundation for these ideas. This foundation uses the algebraic structure of groupoids (see Brandt [1] and Higgins [10]) and greatly generalizes the uses of symmetry in coupled cell systems that we have explored previously $[7,8]$.

Our conventions do not rule out "two-way" coupling, in which cells A and B both influence each other. We represent such a state of affairs by having A coupled to B and B coupled to A. We also do not rule out coupling where cells A and B both influence cell C. Here we consider both $\mathrm{A}$ and $\mathrm{B}$ as being coupled to $\mathrm{C}$. We do not assume the effects of $\mathrm{A}$ and $\mathrm{B}$ to be additive; in fact, the time-evolution of cell $\mathrm{C}$ can in principle be any (smooth) function of the states of $\mathrm{C}, \mathrm{A}$, and $\mathrm{B}$.

In this paper we develop an abstract formalism for coupled cell systems, using simple examples that have no particular role in applications, but it is worth noting that coupled cell systems are used to model a variety of physically interesting systems. For examples, see [8] and references therein. We intend to develop applications of the formalism derived here in future work.

In this section we illustrate some central issues by discussing several examples.

Two-cell systems. We begin with the simplest system of two identical cells (with coordinates $x_{1}$ and $x_{2}$ in $\mathbf{R}^{k}$ ). Without making any specific assumption of the form of the "internal dynamics" of each cell or the form of the "coupling between cells," the differential equations for the coupled system have the form

$$
\begin{aligned}
& \dot{x}_{1}=f\left(x_{1}, x_{2}\right), \\
& \dot{x}_{2}=f\left(x_{2}, x_{1}\right) ;
\end{aligned}
$$

that is, the same function $f$ governs the dynamics of both cells. There are three issues that we discuss concerning system (1.1): the graph (diagram, network) associated to a coupled cell system, symmetry, and synchrony.

Informally, the "network" of a coupled cell system is a finite directed graph whose nodes represent cells and whose edges represent couplings. Nodes are labeled to indicate "equivalent" cells, which have the same phase space and the same internal dynamic. Edges are labeled to indicate "equivalent" couplings. The graph associated to system (1.1) is given in Figure 1. We think of this graph as representing a pair of systems of differential equations in the following way. The two cells are indicated by identical symbols - so they have the same state variables. That is, the coordinates $x_{1}$ of cell 1 and $x_{2}$ of cell 2 lie in the same phase space $\mathbf{R}^{k}$. Since we can interchange cells 1 and 2 without changing the graph, we assume that the same is true for the system of differential equations and that they must have the form (1.1). Note that for this interchange to work, the arrow $1 \rightarrow 2$ must be the same as the arrow $2 \rightarrow 1$.

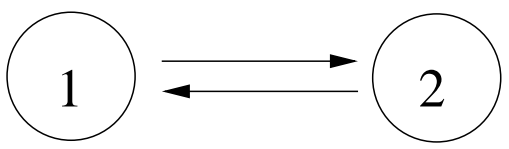

Figure 1. A two-cell network. 
The discussion in the previous paragraph can be summarized by the following: the permutation $\sigma\left(x_{1}, x_{2}\right)=\left(x_{2}, x_{1}\right)$ is a symmetry of the system (1.1). Indeed, more is true: every system of differential equations on $\mathbf{R}^{k} \times \mathbf{R}^{k}$ that is equivariant with respect to $\sigma$ has the form (1.1). That is, abstractly the study of pairs of identical cells that are identically coupled is the same as the study of $\sigma$-equivariant systems. Two consequences follow from this remark. First, synchrony in two-cell systems (solutions such that $x_{1}(t)=x_{2}(t)$ for all time $t$ ) is a robust phenomenon and should not be viewed as surprising. Second, time-periodic solutions can exhibit a kind of generalized synchrony in which the two cells oscillate a half-period out of phase.

The first remark can be restated as follows: the diagonal subspace $V=\left\{x_{1}=x_{2}\right\} \subset$ $\mathbf{R}^{k} \times \mathbf{R}^{k}$ is flow-invariant for every system (1.1). This remark can be verified in two ways. By inspection restrict (1.1) to $V$, obtaining

$$
\begin{aligned}
& \dot{x}_{1}=f\left(x_{1}, x_{1}\right), \\
& \dot{x}_{1}=f\left(x_{1}, x_{1}\right) .
\end{aligned}
$$

It follows that if the initial conditions for a solution satisfy $x_{1}(0)=x_{2}(0)$, then $x_{1}(t)=x_{2}(t)$ for all time $t$, and $V$ is flow-invariant. Alternately, we can observe that $V$ is the fixed-point subspace $\operatorname{Fix}(\sigma)$, and fixed-point subspaces are well known to be flow-invariant.

The second remark is related to general theorems about spatio-temporal symmetries of time-periodic solutions to symmetric systems of ODEs. There are two types of theorems here: existence theorems, asserting that certain spatio-temporal symmetries are possible, and bifurcation theorems, describing particular scenarios that can generate such solutions. The $H / K$ theorem $[4,7]$ is an existence theorem; indeed, it states necessary and sufficient conditions for periodic solutions with a given spatio-temporal symmetry group to be possible. In particular, it implies the existence of functions $f$ having time-periodic solutions of period $T$ satisfying

$$
x_{2}(t)=x_{1}(t+T / 2)
$$

as long as the phase space of each cell has dimension $k \geq 2$. So states with this type of spatio-temporal pattern can exist. Indeed, they can exist robustly (that is, they can persist when $f$ is perturbed) and are therefore typical in the appropriate coupled cell systems. In this case, we can say more: such solutions can arise through Hopf bifurcation. This is a consequence of the general theory of symmetric Hopf bifurcation, [7, 8, 9]. (Note that when $k=1$, nonconstant periodic solutions satisfying (1.2) must intersect the diagonal $V$ and hence be in $V$ for all time: this is a contradiction.)

A three-cell network. Consider the three-cell network illustrated in Figure 2. The systems of differential equations corresponding to this network have the form

$$
\begin{aligned}
& \dot{x}_{1}=f\left(x_{1}, x_{2}\right), \\
& \dot{x}_{2}=g\left(x_{2}, x_{1}, x_{3}\right), \\
& \dot{x}_{3}=f\left(x_{3}, x_{2}\right),
\end{aligned}
$$

where $g\left(x_{2}, x_{1}, x_{3}\right)=g\left(x_{2}, x_{3}, x_{1}\right), x_{1}, x_{3} \in \mathbf{R}^{k}$, and $x_{2} \in \mathbf{R}^{\ell}$. Note that all such systems are equivariant with respect to the permutation $\tau\left(x_{1}, x_{2}, x_{3}\right)=\left(x_{3}, x_{2}, x_{1}\right)$ and that synchronous 


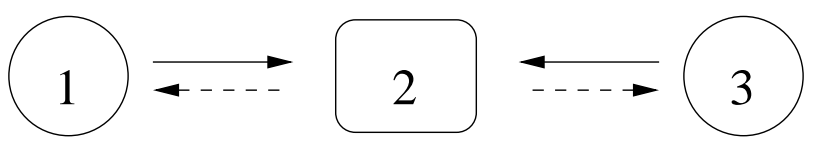

Figure 2. A three-cell network with transposition symmetry.

solutions (where $x_{1}(t)=x_{3}(t)$ for all time $t$ ) occur robustly because the "polydiagonal" subspace $W=\left\{x: x_{1}=x_{3}\right\}$ is flow-invariant for (1.3).

There are two differences between the three-cell network in Figure 2 and the two-cell network in Figure 1. First, not all $\tau$-equivariant systems on $\mathbf{R}^{k} \times \mathbf{R}^{\ell} \times \mathbf{R}^{k}$ have the form (1.3), since in the general $\tau$-equivariant system $f$ can depend nontrivially on both $x_{1}$ and $x_{3}$. So there can be additional structure in coupled cell systems that does not correspond directly to symmetry. Second, the half-period, out of phase, time-periodic solutions satisfy

$$
x_{3}(t)=x_{1}(t+T / 2) \quad \text { and } \quad x_{2}(t)=x_{2}(t+T / 2) .
$$

In particular, the oscillations in cell 2 are forced by symmetry to occur at twice the frequency of those in cells 1 and 3. So multirhythms [7] can be forced by the architecture of coupled cell networks.

Another three-cell network. We now show that robust synchrony is possible in networks that have no symmetry. Consider the three-cell network in Figure 3. Here we have used two distinct symbols (square and circle) for cells and three types of arrows for couplings. The role of these symbols can be seen in the form of the ODE: identical symbols correspond to identical functions in the appropriate variables.

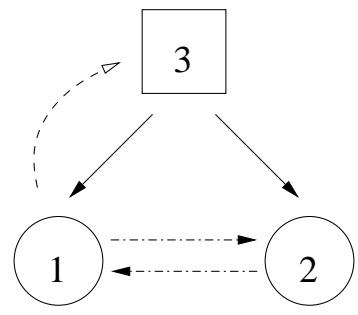

Figure 3. A three-cell network without symmetry.

This network has no symmetry, but the network structure forces the "polydiagonal" subspace $Y=\left\{x: x_{1}=x_{2}\right\}$ to be flow-invariant. To verify this point observe that the coupled cell systems associated with this network have the form

$$
\begin{aligned}
& \dot{x}_{1}=f\left(x_{1}, x_{2}, x_{3}\right), \\
& \dot{x}_{2}=f\left(x_{2}, x_{1}, x_{3}\right), \\
& \dot{x}_{3}=g\left(x_{3}, x_{1}\right),
\end{aligned}
$$

where $x_{1}, x_{2} \in \mathbf{R}^{k}$ and $x_{3} \in \mathbf{R}^{\ell}$. Restricting the first two equations to $Y$ yields

$$
\begin{aligned}
& \dot{x}_{1}=f\left(x_{1}, x_{1}, x_{3}\right), \\
& \dot{x}_{2}=f\left(x_{1}, x_{1}, x_{3}\right),
\end{aligned}
$$


implying that $Y$ is a flow-invariant subspace.

There is a precise sense in which cells 1 and 2 are equivalent within this network, and it is this observation that will enable us to prove the flow-invariance of subspaces like $Y$ in a more abstract (and general) setting. Define the "input set" of a cell $j$ to be the cell $j$ and all cells $i$ that connect to cell $j$. Also include the arrows from cells $i$ to $j$. See Figure 4 .

We can now explain why $Y$ is flow-invariant, in terms of a permutation that acts on the network. This permutation is not a symmetry of the whole network, but it preserves enough structure to create a flow-invariant subspace. The key property is that the input sets of cells 1 and 2 are isomorphic via the permutation $\sigma$ that maps $\left(\begin{array}{llll}1 & 2 & 3\end{array}\right) \rightarrow\left(\begin{array}{lll}2 & 1 & 3\end{array}\right)$.

If the system (1.5) were equivariant with respect to $\sigma$, then the fixed-point space of $\sigma$ would be flow-invariant by [9, 7]. Moreover, the fixed-point space of $\sigma$ is $Y$. However, (1.5) is not equivariant with respect to $\sigma$. Indeed, if we apply $\sigma$, then the equation transforms into

$$
\begin{aligned}
& \dot{x}_{2}=f\left(x_{2}, x_{1}, x_{3}\right), \\
& \dot{x}_{1}=f\left(x_{1}, x_{2}, x_{3}\right), \\
& \dot{x}_{3}=g\left(x_{3}, x_{2}\right) .
\end{aligned}
$$

The first two equations are the same as in (1.5), but the third equation is not. However, the third is the same on the space $Y$, where $x_{2}=x_{1}$. So the restriction of the equations to $Y$ is $\sigma$-equivariant, and this is enough to make $Y$ flow-invariant.

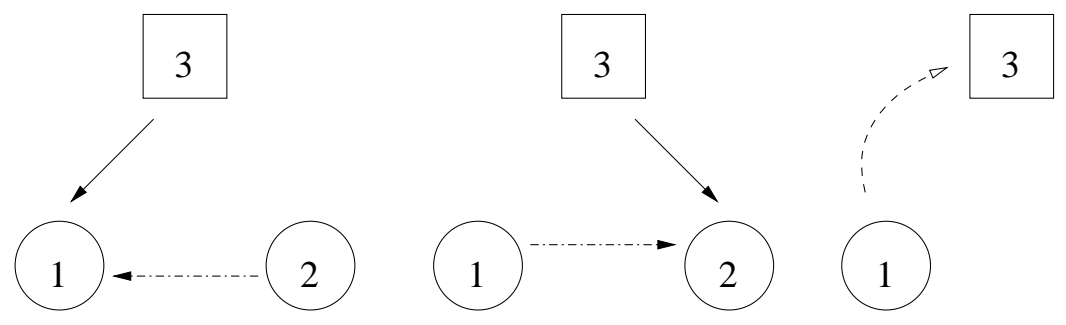

Figure 4. Input sets for three-cell network without symmetry.

Traveling waves in a seven-cell network. Consider the seven-cell linear network in Figure 5. The corresponding differential equations have the general form

$$
\begin{aligned}
& \dot{x}_{1}=B\left(x_{1}\right), \quad \dot{x}_{2}=A\left(x_{2}, x_{1}\right), \quad \dot{x}_{3}=A\left(x_{3}, x_{2}\right), \\
& \dot{x}_{4}=A\left(x_{4}, x_{3}\right), \quad \dot{x}_{5}=A\left(x_{5}, x_{4}\right), \quad \dot{x}_{6}=A\left(x_{6}, x_{5}\right) \text {, } \\
& \dot{x}_{7}=A\left(x_{7}, x_{6}\right) \text {. }
\end{aligned}
$$

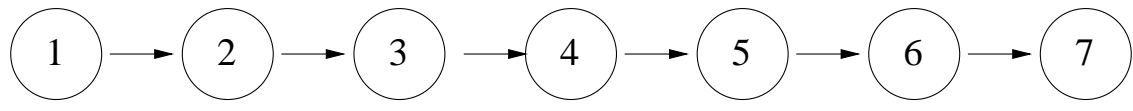

Figure 5. Seven-cell linear network.

It does not seem to be a simple matter to determine whether traveling waves are present in this network. If the cell phase spaces are all one-dimensional, there are no nontrivial 
time-periodic states so no traveling waves. With higher-dimensional phase spaces, special assumptions are needed to produce traveling waves. However, if we introduce back coupling from cell 3 to cell 1, as shown in Figure 6, traveling waves can typically be expected, even in the one-dimensional case, as explained below. This is curious, because informally Figure 6 would normally be considered as being less regular in form than Figure 5. So the issue of "regular form" for a coupled cell network is fairly subtle. The key feature here is that all input sets for cells in the network in Figure 6 are isomorphic, whereas this is not true for the cells in Figure 5. It is this additional "symmetry" on the groupoid level that makes traveling waves typical. Indeed, Figure 6 has many groupoid symmetries (42 in all).

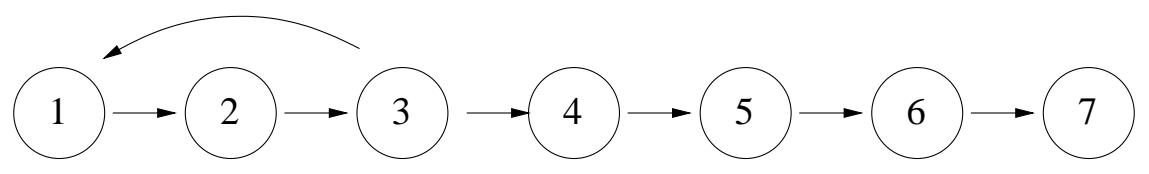

Figure 6. Seven-cell linear network with back connection.

We discuss why traveling wave solutions arise in two ways. First, the assumption that all of the cells and arrows in Figure 6 are identical implies that the first equation in (1.7) is now

$$
\dot{x}_{1}=A\left(x_{1}, x_{3}\right) \text {. }
$$

If we set

$$
\begin{aligned}
& x_{7}=x_{4}=x_{1}=y_{1}, \\
& x_{6}=x_{3}=y_{3}, \\
& x_{5}=x_{2}=y_{2},
\end{aligned}
$$

then the system of seven equations reduces to a three-equation system

$$
\begin{aligned}
& \dot{y}_{1}=A\left(y_{1}, y_{3}\right), \\
& \dot{y}_{2}=A\left(y_{2}, y_{1}\right), \\
& \dot{y}_{3}=A\left(y_{3}, y_{2}\right),
\end{aligned}
$$

which is the general form associated with the directed ring of coupled cells in Figure 7 . It is not hard to show using Hopf bifurcation (see [7,9]) that the system (1.9) can support a discrete rotating wave $y(t)$, where

$$
y_{2}(t)=y_{1}\left(t-\frac{T}{3}\right), \quad y_{3}(t)=y_{2}\left(t-\frac{T}{3}\right)
$$

and $y_{1}$ is periodic of period $T$. This solution yields a traveling wave solution for the network in Figure 6. See a sample simulation in Figure 8. (The number 7 is not significant here: the same ideas work for any chain containing three or more cells and with feedback from any cell other than the first.)

More importantly, the three-cell ring in Figure 7 is a quotient network of the one in Figure 6 , where the quotient map $\beta$ takes $x_{1}, x_{4}, x_{7}$ to $y_{1}, x_{2}, x_{5}$ to $y_{2}$, and $x_{3}, x_{6}$ to $y_{3}$. We define "quotient" in section 8, but the key point is that solutions for the three-cell ring 


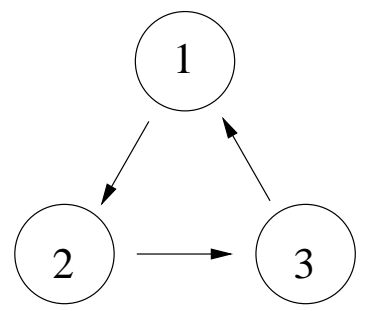

Figure 7. Three-cell directed ring: Quotient of the network in Figure 6.

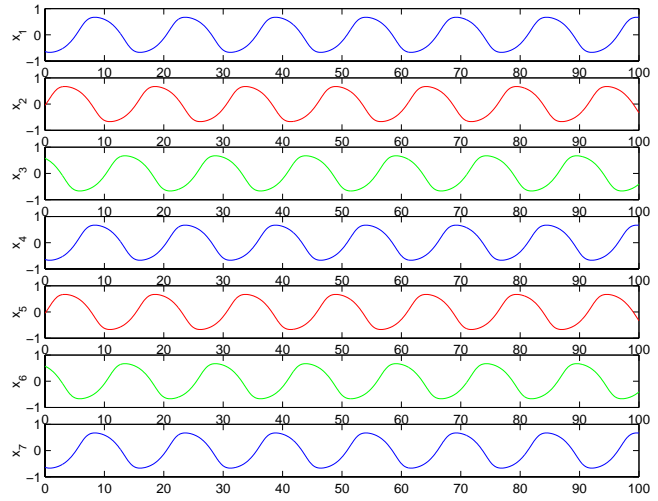

Figure 8. Traveling wave solution in seven-cell chain.

naturally "lift" to solutions for the seven-cell network via (1.8). The crucial features here are that $\beta$ induces an isomorphism from each input set in the first network to an input set in the second network, and every coupled cell system of differential equations in the quotient lifts to a coupled cell system in the first network.

We seek to isolate the abstract structural features that are responsible for the behavior described in the above examples and to place the discussion in a rigorous formal context. We structure the paper as follows. Coupled cell networks are rigorously defined in terms of nodes and arrows in section 2. The key concept, the groupoid structure of a coupled cell network, is defined in section 3 in terms of input sets. The phase space and admissible vector fields associated to a coupled cell network are discussed in section 4. Basically, the intuitive ideas presented in this introduction are formalized as equivariance with respect to the symmetry groupoid of the network. Section 5 describes an extended example, which motivates the rest of the paper. In section 6 we introduce two different notions of robust synchrony: flow-invariant subspaces and balanced equivalence relations. We prove that these notions are equivalent. Quotient maps and quotient networks, which constitute a fourth equivalent notion for synchrony, are discussed in section 8. Quotient networks are an especially useful concept because they illuminate the generic dynamics of vector fields restricted to synchronous invariant subspaces, which can include phase-locked states and synchronized chaos. Examples illustrating these points are discussed in section 7 . The relationship between the dynamics on a synchronous subspace and the induced dynamics on the quotient network is discussed in section 9 . 
2. Coupled cell networks. We begin by formally defining a coupled cell network. At this stage we consider only the abstract network architecture (a labeled directed graph). The associated family of ODEs is discussed in section 4.

Definition 2.1. A coupled cell network $G$ consists of the following:

(a) A finite set $\mathcal{C}=\{1, \ldots, N\}$ of nodes or cells.

(b) A finite set of ordered pairs $\mathcal{E} \subseteq \mathcal{C} \times \mathcal{C}$ of directed edges or arrows. Each edge $(c, d)$ has $a$ tail $c$ and $a$ head $d$.

(c) An equivalence relation $\sim_{C}$ on cells in $\mathcal{C}$. The type or cell label of cell c is the $\sim_{C^{-}}$ equivalence class $[c]_{C}$ of $c$.

(d) An equivalence relation $\sim_{E}$ on edges in $\mathcal{E}$. The type or coupling label of edge $e$ is the $\sim_{E}$-equivalence class $[e]_{E}$ of $e$. An edge $(c, c)$ is an internal edge; a cell is active if it has an internal edge. We assume that every cell is active, that is,

$$
\Delta_{\mathcal{C}}=\{(c, c): c \in \mathcal{C}\} \subset \mathcal{E} .
$$

In addition, we require the following compatibility conditions:

(e) Equivalent edges have equivalent tails and heads. That is, if $(i, c) \sim_{E}(j, d)$, then $i \sim_{C} j$ and $c \sim_{C} d$.

(f) Internal edges are equivalent if their tails are equivalent. Internal edges and noninternal edges are never equivalent. That is, for all $c, d, d^{\prime} \in \mathcal{C}$,

$$
(c, c) \sim_{E}\left(d, d^{\prime}\right) \Longleftrightarrow d=d^{\prime} \text { and } d \sim_{C} c .
$$

Formally, the coupled cell network $G$ is the quadruple $G=\left(\mathcal{C}, \mathcal{E}, \sim_{C}, \sim_{E}\right)$.

We represent a coupled cell network $G$ by a diagram constructed as follows.

(1) For each $\sim_{C}$-equivalence class of cells choose a distinct node symbol $\bigcirc, \square, \triangle$, and so on.

(2) For each $\sim_{E}$-equivalence class of noninternal edges, choose a distinct arrow $\rightarrow, \Rightarrow, \sim$, and so on.

The compatibility conditions in Definition 2.1 state that arrows between distinct cells can be identical only when the nodes at the heads are identical and the nodes at the tails are identical, and that node symbols can be interpreted as arrows from a cell to itself.

The above definition is essentially the standard concept of a directed graph (or digraph) in graph theory (see, for example, Tutte [13], Wilson [16]) modified to incorporate labeling of nodes and edges. We assume that the graph is finite because this makes the associated dynamical systems (discussed in section 4) finite-dimensional. However, most of the theory generalizes to infinite graphs. The assumption that all cells are active can be removed, at the expense of notational complications, but the details are routine and we do not treat this case here.

Example 2.2. Suppose that the network $G$ is defined by

$$
\begin{aligned}
& \mathcal{C}=\{1,2,3,4\}, \\
\mathcal{E} & =\{(1,2),(1,3),(2,4),(3,1),(3,4)(4,1)\}, \\
\sim_{C} & \text { has equivalence classes }\{1\},\{2,3\},\{4\}, \\
\sim_{E} & \text { has equivalence classes }\{(1,2),(1,3)\},\{(2,4),(3,4)\},\{(3,1)\},\{(4,1)\},
\end{aligned}
$$


and all nodes are active. Then the diagram of $G$ has the form shown in Figure 9 for the given choices of symbols.

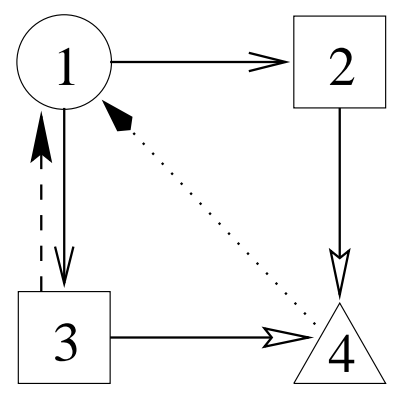

Figure 9. Example of the diagram of a coupled cell network.

3. Input sets and groupoids. In this section we define the basic algebraic structure of a coupled cell network - its symmetry groupoid. Some preliminary concepts are required.

Input sets. As discussed in more detail in section 4 the variables that appear in a given component $f_{c}$ of the vector fields $f$ associated to a coupled cell network depend only on those cells that are linked to cell $c$ by an arrow. This observation is abstracted as the following definition.

Definition 3.1. The input set $I(c)$ of a cell $c$ is

$$
I(c)=\{i \in \mathcal{C}:(i, c) \in \mathcal{E}\} .
$$

Since all cells are active, $c \in I(c)$. We call $c$ the base cell of $I(c)$.

Two cells with isomorphic input sets will be called "input equivalent."

Definition 3.2. The relation $\sim_{I}$ of input equivalence on $\mathcal{C}$ is defined by $c \sim_{I} d$ if and only if there exists a base cell preserving bijection

$$
\beta: I(c) \rightarrow I(d)
$$

(by which we mean that $\beta(c)=d$ ) such that for all $i \in I(c)$

$$
(i, c) \sim_{E}(\beta(i), d)
$$

Any such bijection $\beta$ is called an input isomorphism from cell $c$ to cell $d$. The set $B(c, d)$ denotes the collection of all input isomorphisms from cell $c$ to cell $d$.

On setting $i=c$ in (3.2), we see that $c$ and $d$ have the same type $\left(c \sim_{C} d\right)$ if they are input equivalent $\left(c \sim_{I} d\right)$. The converse is easily seen to be false. Moreover, $B(c, d)$ is empty unless $c \sim{ }_{I} d$.

An important class of networks is one in which all cells are input equivalent. So we define the following.

Definition 3.3. A homogeneous network is a coupled cell network such that $B(c, d) \neq \emptyset$ for every pair of cells $c, d$.

Examples of homogeneous networks are given in Figures 14 and 15. 
Example 3.4. We return to Example 2.2 whose diagram is shown in Figure 9. The input

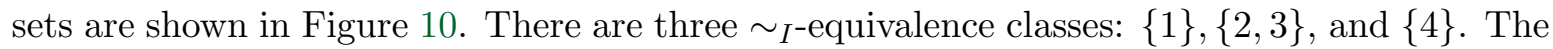
isomorphism between $I(2)$ and $I(3)$ is the bijection $\tau:\{2,1\} \rightarrow\{3,1\}$ for which $\tau(2)=3$ and $\tau(1)=1$.
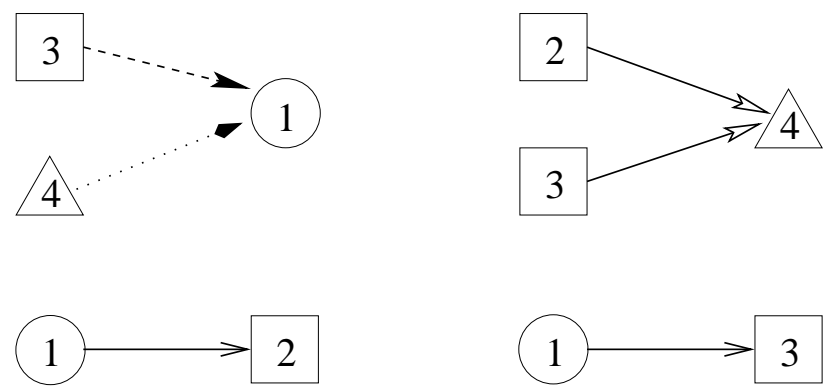

Figure 10. Input sets for Figure 9.

The symmetry groupoid. We now introduce the central concept of this paper, the "symmetry groupoid" of a coupled cell network. The symmetry groupoid is a generalization of the symmetry group of a symmetric network. It includes not just symmetries of the whole network, but symmetries between particular subgraphs - namely, the input sets.

Definition 3.5. The symmetry groupoid of a coupled cell network $G$ is the disjoint union

$$
\mathcal{B}_{G}=\bigcup_{c, d \in \mathcal{C}} B(c, d) \text {. }
$$

The term "groupoid" was introduced by Brandt [1] and is developed at length in Higgins [10]. The term refers to an algebraic structure that is similar to a group, with the exception that products of elements may not always be defined. Different authors formalize groupoids in slightly different (but mostly equivalent) ways. Essentially, a groupoid must satisfy three conditions:

(1) The product operation is associative in the sense that whenever one of $\alpha(\beta \gamma)$ and $(\alpha \beta) \gamma$ is defined, then so is the other, and they are equal.

(2) There are distinguished elements $\varepsilon_{j}$ that act as identity elements, in the sense that $\varepsilon_{j} \alpha=\alpha$ and $\alpha \varepsilon_{j}=\alpha$ whenever these are defined. (Here the indices $j$ correspond to the "objects" of the groupoid, which in our case are the cells.)

(3) Every element $\alpha$ has an inverse $\alpha^{-1}$, in the sense that both $\alpha \alpha^{-1}$ and $\alpha^{-1} \alpha$ are identities.

In the case of $\mathcal{B}_{G}$, the groupoid structure is captured by the following:

(1) We define the product of $\beta_{1} \in B(c, d)$ and $\beta_{2} \in B\left(c^{\prime}, d^{\prime}\right)$ if and only if $c^{\prime}=d$, and then we set $\beta_{2} \beta_{1}=\beta_{2} \circ \beta_{1} \in B\left(c, d^{\prime}\right)$, where $\circ$ denotes composition of maps. Composition is of course associative when it is defined.

(2) The identity elements $\operatorname{id}_{I(c)}$ for $c \in \mathcal{C}$ are the groupoid identity elements.

(3) For inverses, observe that $\beta \in B(c, d)$ if and only if $\beta^{-1} \in B(d, c)$.

It follows in particular that $B(c, c)$ is a group, the vertex group corresponding to $c$. Vertex groups are important in groupoid theory and play a key role in this paper. 
Remark 3.6. The term "disjoint union" in Definition 3.5 is used in a technical sense. The sets $B(c, d)$ for different pairs $(c, d)$ are not necessarily disjoint. For example, if $\mathcal{C}=\{1,2\}$, where cells 1 and 2 are inequivalent, each coupled to the other by inequivalent arrows, then $B(1,1)$ and $B(2,2)$ both consist of the identity map on $\{1,2\}$. It is convenient to make them disjoint. One way to do this is to replace each bijection $\beta \in B(c, d)$ by the triple $(\beta, c, d)$. Then $\beta$ defines the permutation, $c$ is an index specifying its "domain," and $d$ is an index specifying its "range." As far as the groupoid structure is concerned, the product $\beta_{2} \beta_{1}$ is defined only when $c^{\prime}=d$. This occurs when the "range" of $\beta_{1}$ is equal to the "domain" of $\beta_{2}$, in the sense just specified. However, the set-theoretic ranges and domains of the corresponding bijections may permit the composition of $\beta_{1}$ and $\beta_{2}$ as functions in cases where we do not wish to permit them to be multiplied in the groupoid.

The point here is that we are not dealing merely with bijections on sets but with base point preserving bijections on based sets. Composition must respect the base points as well as the sets.

For simplicity, we use $\beta$ to denote an input isomorphism, rather than the cumbersome $(\beta, c, d)$, because the appropriate $c, d$ are usually obvious.

Alternatively, it would be possible to represent the input structure of a given cell as consisting of that cell, all cells connected to it, and the associated arrows. Input isomorphisms then necessarily preserve the base point (the cell at the head of all arrows). Technically, however, this choice causes other complications - for example, input isomorphisms now act on subgraphs and not on subsets of cells - so we shall not use it here. However, it is a useful informal way to visualize input isomorphisms.

Example 3.7. Again, we return to Example 2.2. The nonempty sets $B(c, d)$ are as follows:

$B(1,1)$ : The identity map on $\{1,3,4\}$.

$B(2,2)$ : The identity map on $\{2,1\}$.

$B(3,3)$ : The identity map on $\{3,1\}$.

$B(4,4)$ : The identity map on $\{4,2,3\}$ and the permutation $\sigma$ on $\{4,2,3\}$ for which $\sigma(2)=$ $3, \sigma(3)=2, \sigma(4)=4$.

$B(2,3):$ The map $\tau:\{2,1\} \rightarrow\{3,1\}$ for which $\tau(1)=1, \tau(2)=3$.

$B(3,2)$ : The inverse $\tau^{-1}$ of $\tau$.

Subgroupoids and connected components. For the basics of groupoids see Brandt [1], Brown [3], Higgins [10], and MacLane [11]. For applications see Weinstein [15]. Groupoids combine several features of groups with features of graphs, and we discuss one of each now. The group-theoretic notion is that of a subgroupoid; the graph-theoretic one is that of a connected component.

A subset $\mathcal{S} \subset \mathcal{B}_{G}$ is a subgroupoid if $\mathcal{S}$ is closed under products (when defined) and taking inverses.

The connected components of the groupoid $\mathcal{B}_{G}$ are in one-to-one correspondence with $\sim_{I^{-}}$

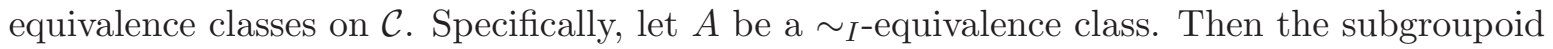

$$
\mathcal{S}(A)=\bigcup_{c, d \in A} B(c, d)
$$

is a connected component of $\mathcal{B}_{G}$. Moreover, we have the following lemma. 
Lemma 3.8. The groupoid $\mathcal{B}_{G}$ is the disjoint union of its connected components. That is,

$$
\mathcal{B}_{G}=\bigcup_{A} \mathcal{S}(A)
$$

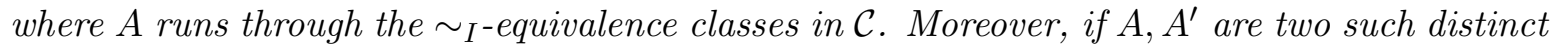
classes, and $\beta \in \mathcal{S}(A), \beta^{\prime} \in \mathcal{S}\left(A^{\prime}\right)$, then the product $\beta \beta^{\prime}$ is not defined.

We say that two cells $c, d \in \mathcal{C}$ are in the same connected component of $\mathcal{B}_{G}$ if and only if $c \sim_{I} d$. The mental image here is that associated with any groupoid there is a graph, whose elements are the vertices of the groupoid and whose (directed) edges are the groupoid elements. It is the connected components of this graph that are being described. See Higgins [10, Chapter 3].

If $c, d$ belong to the same connected component, then the vertex groups $B(c, c)$ and $B(d, d)$ are conjugate, in the sense that there exists $\gamma \in B(c, d)$ such that

$$
B(c, c)=\gamma^{-1} B(d, d) \gamma .
$$

In particular, $B(c, c)$ and $B(d, d)$ are isomorphic groups.

Structure of $\boldsymbol{B}(c, d)$. For later use, we determine the general structure of the sets $B(c, d)$.

(1) If $c \chi_{I} d$, then $B(c, d)=\emptyset$.

(2) If $c=d$, then we define an equivalence relation $\equiv_{c}$ on $I(c)$ by

$$
j_{1} \equiv_{c} j_{2} \Longleftrightarrow\left(j_{1}, c\right) \sim_{E}\left(j_{2}, c\right)
$$

for $j_{1}, j_{2} \in I(c)$. Let the $\equiv_{c^{-}}$equivalence classes of $I(c)$ be $K_{0}, \ldots, K_{r}$ for $r=r(c)$ so that

$$
I(c)=K_{0} \dot{\cup} \cdots \dot{\cup} K_{r} .
$$

We may choose $K_{0}=\{c\}$ by Definition 2.1(f). Let

$$
k_{s}=\left|K_{s}\right| \quad(0 \leq s \leq r) .
$$

Then $B(c, c)$ is a group given by

$$
B(c, c)=\mathbf{S}_{k_{1}} \times \cdots \times \mathbf{S}_{k_{r}},
$$

where each $\mathbf{S}_{k_{s}}$ comprises all permutations of $K_{s}$, extended by the identity on $I(c) \backslash K_{s}$. (3) If $c \sim_{I} d$ and $c \neq d$, define $\equiv_{d}$ on $I(d)$ in the same way. Let $\beta \in B(c, d)$, and let

$$
L_{s}=\beta\left(K_{s}\right) \quad(0 \leq s \leq r(c)) .
$$

Then $\beta$ and $\beta^{-1}$ preserve $\sim_{E}$, so the $\equiv_{d^{-}}$equivalence classes of $I(d)$ are the $L_{s}(0 \leq s \leq$ $r(c))$, and $r(d)=r(c)$.

Choose a fixed but arbitrary $\beta_{0} \in B(c, d)$, having the above property. Then

$$
B(c, d)=B(d, d) \beta_{0}=\beta_{0} B(c, c) .
$$

Conversely, any $\beta_{0}: I(c) \rightarrow I(d)$ such that $L_{s}=\beta_{0}\left(K_{s}\right)$ for $0 \leq s \leq r(c)$ lies in $B(c, d)$. 
4. Vector fields on a coupled cell network. We now define the class $\mathcal{F}_{G}^{P}$ of vector fields corresponding to a given coupled cell network $G$. This class consists of all vector fields that are "compatible" with the labeled graph structure or, equivalently, are "symmetric" under the groupoid $\mathcal{B}_{G}$. It also depends on a choice of "total phase space" $P$, which we assume is fixed throughout the subsequent discussion. For example, in the two-cell system (1.1) we have $P=\mathbf{R}^{k} \times \mathbf{R}^{k}$, which depends on the choice of $k$.

For each cell in $\mathcal{C}$ define a cell phase space $P_{c}$. This must be a smooth manifold of dimension $\geq 1$, which for simplicity we assume is a nonzero finite-dimensional real vector space. We require

$$
c \sim_{C} d \quad \Longrightarrow \quad P_{c}=P_{d}
$$

and we employ the same coordinate systems on $P_{c}$ and $P_{d}$. Only these identifications are canonical. If $P_{c}=P_{d}$ or $P_{c}$ is isomorphic to $P_{d}$ when $c \chi_{C} d$, then the identification of $P_{c}$ and $P_{d}$ will be deemed accidental and will have no significance for our present purposes. Although the relation $c \sim_{C} d$ means that cells $c$ and $d$ have the same phase space, it does not imply that they have isomorphic (that is, conjugate) dynamics.

Define the corresponding total phase space to be

$$
P=\prod_{c \in \mathcal{C}} P_{c}
$$

and employ the coordinate system

$$
x=\left(x_{c}\right)_{c \in \mathcal{C}}
$$

on $P$.

The cell projection corresponding to cell $c$ is the natural projection

$$
\pi_{c}: P \rightarrow P_{c}
$$

More generally, suppose that $\mathcal{D}$ is any subset of $\mathcal{C}$. Define

$$
P_{\mathcal{D}}=\prod_{c \in \mathcal{D}} P_{c}
$$

and let

$$
\pi_{\mathcal{D}}: P \rightarrow P_{\mathcal{D}}
$$

be the natural projection. Further, write

$$
x_{\mathcal{D}}=\pi_{\mathcal{D}}(x)
$$

and suppress braces when $\mathcal{D}$ is a singleton. That is, $\pi_{c}(x)=x_{c}=x_{\{c\}}$.

Finally, suppose that $\mathcal{D}_{1}, \mathcal{D}_{2}$ are subsets of $\mathcal{C}$ and that there is a bijection $\beta: \mathcal{D}_{1} \rightarrow \mathcal{D}_{2}$ such that $\beta(d) \sim_{C} d$ for all $d \in \mathcal{D}_{1}$. Define the pullback map

$$
\beta^{*}: P_{\mathcal{D}_{2}} \rightarrow P_{\mathcal{D}_{1}}
$$


by

$$
\left(\beta^{*}(z)\right)_{j}=z_{\beta(j)} \quad \forall j \in \mathcal{D}_{1}, z \in P_{\mathcal{D}_{2}} .
$$

By direct calculation it is easy to verify three simple properties of the pullback:

$$
\begin{aligned}
(\beta \gamma)^{*} & =\gamma^{*} \beta^{*} \\
\mathrm{id}^{*} & =\mathrm{id} \\
\left(\gamma^{-1}\right)^{*} & =\left(\gamma^{*}\right)^{-1}
\end{aligned}
$$

Note the reversed order in the first of these equations.

We use pullback maps to relate different components of the vector field associated with a given coupled cell network. Specifically, the class of vector fields that is encoded by a coupled cell network will be defined using the following concept.

Definition 4.1. A vector field $f: P \rightarrow P$ is $\mathcal{B}_{G}$-equivariant or $G$-admissible if the following hold:

(a) For all $c \in \mathcal{C}$ the component $f_{c}(x)$ depends only on $x_{I(c)}$; that is, there exists $\hat{f}_{c}$ : $P_{I(c)} \rightarrow P_{c}$ such that

$$
f_{c}(x)=\hat{f}_{c}\left(x_{I(c)}\right)
$$

(b) For all $c, d \in \mathcal{C}$ and $\beta \in B(c, d)$ (so that, in particular, $d=\beta(c)$ )

$$
\hat{f}_{d}\left(x_{I(d)}\right)=\hat{f}_{c}\left(\beta^{*}\left(x_{I(d)}\right)\right) \quad \forall x \in P .
$$

For brevity, we write this condition as

$$
f_{\beta(c)}(x)=f_{c}\left(\beta^{*}(x)\right) \quad \forall x \in P .
$$

When using (4.5) it is necessary to bear in mind the constraint that $f_{d}(x)$ depends only on $x_{I(d)}$. Otherwise, $\beta^{*}(x)$ is not defined.

We call (a) the domain condition and (b) the equivariance condition on $f$.

Remark 4.2. If $\beta$ belongs to the vertex group $B(c, c)$, then (4.5) implies that

$$
f_{c}\left(\beta^{*}(x)\right)=f_{c}(x) \quad \forall x \in P .
$$

That is, $f_{c}$ is $B(c, c)$-invariant. It is easy to check that this property is the same as the usual property of invariance under a group, provided we consider $B(c, c)$ as acting on $P_{I(c)}$.

Definition 4.3. For a given choice of the $P_{c}$ we define the class $\mathcal{F}_{G}^{P}$ to consist of all $G$ admissible vector fields on $P$.

These are the most general vector fields on $P$ that are consistent with the coupled cell network.

Example 4.4. We describe $\mathcal{F}_{G}^{P}$ for the diagram of Figure 9. There are three cell types $\bigcirc, \square, \triangle$, and we choose three corresponding phase spaces $U, V, W$. Then the state variable is 
$x=\left(x_{1}, x_{2}, x_{3}, x_{4}\right)$, where $x_{1} \in U, x_{2}, x_{3} \in V, x_{4} \in W$. There are four arrow types. We claim that the $G$-admissible vector fields $f$ are those of the form

$$
\begin{aligned}
& f_{1}(x)=A\left(x_{1}, x_{3}, x_{4}\right), \quad \text { where } A: U \times V \times W \rightarrow U, \\
& f_{2}(x)=B\left(x_{2}, x_{1}\right), \quad \text { where } B: V \times U \rightarrow V, \\
& f_{3}(x)=B\left(x_{3}, x_{1}\right),
\end{aligned}
$$

and $C$ is symmetric in $x_{2}, x_{3}$.

To prove this, we consider the equivariance condition (4.5) for all the bijections $\beta$ listed in Example 3.7. There are two nontrivial cases: $B(2,3)$ and $B(4,4)$. First, suppose that $c=2$ andd $=3$, and consider the bijection $\tau: I(2) \rightarrow I(3)$ for which $\tau(2)=3, \tau(1)=1$. Suppose that we define the function $B: P_{I(2)} \rightarrow P_{2}$ by

$$
B\left(x_{2}, x_{1}\right)=f_{2}(x)
$$

so that $B=\hat{f}_{2}$. Then $f_{3}(x)=\hat{f}_{3}\left(x_{3}, x_{1}\right)$, and we wish to express this in terms of $B$.

It is easy to work out the pullback of $\tau$. If we write the elements of $P_{I(3)}$ in the form $x=\left(x_{3}, x_{1}\right)$, then $y=\tau^{*}(x)$ takes the form $y=\left(y_{2}, y_{1}\right) \in P_{I(2)}$, where

$$
\begin{aligned}
& y_{2}=(x)_{\tau(2)}=x_{3}, \\
& y_{1}=(x)_{\tau(1)}=x_{1} .
\end{aligned}
$$

Then

$$
\tau^{*}\left(x_{3}, x_{1}\right)=\left(x_{3}, x_{1}\right),
$$

and condition (4.5) tells us that

$$
\hat{f}_{3}(x)=B\left(x_{3}, x_{1}\right)
$$

as claimed. (The pullback $\tau^{*}$ is not the identity, because its range and domain are different. It is an identification.)

Similarly, if we consider $\sigma \in B(4,4)$, then we have a function $C$ defined by $f_{4}(x)=$ $C\left(x_{4}, x_{2}, x_{3}\right)$. Now the pullback $\sigma^{*}: P_{I(4)} \rightarrow P_{I(4)}$ acts as

$$
\sigma^{*}\left(x_{4}, x_{2}, x_{3}\right)=\left(x_{4}, x_{3}, x_{2}\right)
$$

and condition (4.5) tells us that

$$
C\left(x_{4}, x_{2}, x_{3}\right)=\hat{f}_{4}(x)=C\left(x_{4}, x_{3}, x_{2}\right)
$$

so that $C$ is symmetric in $x_{2}, x_{3}$.

Here and from now on we adopt the convention that $x_{c}$ is the first variable listed in the argument of $\hat{f}_{c}$. We can show that $\hat{f}_{c}$ is symmetric in some subset of variables by putting a bar over that set so that here

$$
f_{4}(x)=C\left(x_{4}, \overline{x_{2}, x_{3}}\right) .
$$

(To do this, we have to order the variables suitably, and in some cases this cannot be done consistently. The use of a bar is convenient for the purposes of this paper.) Note that the network $G$ is not symmetric under the 2-cycle (23), because the arrow from cell 3 to cell 1 does not correspond to an arrow from cell 2 to cell 1. 
Admissible vector fields. The proofs of the main theorems of this paper rely on the construction of certain special $G$-admissible vector fields. In this subsection we describe these constructions.

As motivation, consider Example 4.4. Here, the most general $G$-admissible vector field is specified by three functions $A, B, C$. These functions can be assigned independently of each other. There is one such function for each $\sim_{I^{-}}$equivalence class of cells, that is, each connected component of $\mathcal{B}_{G}$. If $c \in \mathcal{C}$, then $f_{c}$ is $B(c, c)$-invariant; if $d \sim_{I} c$, then $f_{d}$ is uniquely defined by $f_{c}$ through the condition of $\mathcal{B}_{G}$-equivariance. We now give a formal statement of these properties and show that they are valid in general.

The main point is that Lemma 3.8 implies that $\mathcal{B}_{G}$-equivariance imposes conditions relating components $f_{c}, f_{d}$ of $f$ when $c, d$ lie in the same connected component of $\mathcal{B}_{G}$, but not otherwise. We can therefore construct $G$-admissible vector fields $g$ on $P$ whose components $g_{c}$ are zero for all $c$ outside a fixed $\sim_{I}$-equivalence class. We will prove that such vector fields $\operatorname{span} \mathcal{F}_{G}^{P}$.

Let $Q \subseteq \mathcal{C}$ be a $\sim_{I^{-} \text {equivalence class. Define }}$

$$
\mathcal{F}_{G}^{P}(Q)=\left\{f \in \mathcal{F}_{G}^{P}: f_{s}(x)=0 \quad \forall s \notin Q\right\} .
$$

Vector fields in $\mathcal{F}_{G}^{P}(Q)$ are supported on $Q$. The subset $\mathcal{F}_{G}^{P}(Q)$ is a linear subspace of $\mathcal{F}_{G}^{P}$.

The key constraint on a vector field in $\mathcal{F}_{G}^{P}(Q)$ is $B(q, q)$-equivariance for some fixed but arbitrary $q \in Q$. In fact we have the following lemma.

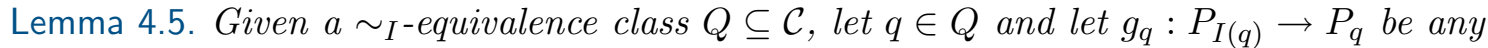
$B(q, q)$-invariant mapping. Then $g_{q}$ extends uniquely to a vector field in $\mathcal{F}_{G}^{P}(Q)$.

Proof. For any $r \in Q$, choose $\beta_{0} \in B(q, r)$ (which exists since $r \sim_{I} q$ ) so that $\beta_{0}(q)=r$. Equivariance forces us to define

$$
g_{r}(y)=g_{q}\left(\beta_{0}^{*}(y)\right) \quad \forall y \in P_{I(r)},
$$

so the extension to $r \in Q$ is unique if it exists. It is easy to show that $g_{r}$ does not depend on the choice of $\beta_{0}$. Finally, if $r \notin Q$, we define $g_{r}(x)=0$.

We have now extended $g_{q}$ to a vector field $g$ on the whole of $P$. We claim that $g \in \mathcal{F}_{G}^{P}(Q)$. Clearly, the components $g_{r}$ of $g$ with $r \notin Q$ vanish. It is therefore sufficient to show that if $r \in Q, \gamma \in B(r, s)$, and $z \in P_{I(s)}$, then

$$
g_{s}(z)=g_{r}\left(\gamma^{*}(z)\right) .
$$

The component $g_{s}$ is defined by choosing $\beta_{1} \in B(q, s)$ and setting

$$
g_{s}(y)=g_{q}\left(\beta_{1}^{*}(y)\right) \quad \forall y \in P_{I(s)} .
$$

To establish (4.9), let

$$
\delta=\beta_{1}^{-1} \gamma \beta_{0} \in B(q, q)
$$

so that

$$
\gamma=\beta_{1} \delta \beta_{0}^{-1}
$$


Then, using (4.2), we compute

$$
\begin{aligned}
g_{r}\left(\gamma^{*}(z)\right) & =g_{r}\left(\left(\beta_{1} \delta \beta_{0}^{-1}\right)^{*}(z)\right) \\
& =g_{r}\left(\left(\beta_{0}^{*}\right)^{-1} \delta^{*} \beta_{1}^{*}(z)\right) \\
& =g_{q}\left(\beta_{0}^{*}\left(\beta_{0}^{*}\right)^{-1} \delta^{*} \beta_{1}^{*}(z)\right) \\
& =g_{q}\left(\delta^{*}\left(\beta_{1}^{*}(z)\right)\right) \\
& =g_{q}\left(\beta_{1}^{*}(z)\right) \\
& =g_{s}(z)
\end{aligned}
$$

(where $g_{q}\left(\delta^{*}\left(\beta_{1}^{*}(z)\right)\right)=g_{q}\left(\beta_{1}^{*}(z)\right)$ because $\beta_{1}^{*}(z) \in I(q)$ and $g_{q}$ is $B(q, q)$-invariant). This calculation proves $(4.9)$.

The importance of such vector fields $g$ stems from the following proposition.

Proposition 4.6.

$$
\mathcal{F}_{G}^{P}=\bigoplus_{Q} \mathcal{F}_{G}^{P}(Q)
$$

where $Q$ runs over the $\sim_{I}$-equivalence classes of $G$.

Proof. Suppose that $f \in \mathcal{F}_{G}^{P}$, so that $f$ is $\mathcal{B}_{G}$-equivariant. Let $Q$ be a $\sim_{I}$-equivalence class, and pick $q \in Q$. Define $g \in \mathcal{F}_{G}^{P}(Q)$ by setting

$$
g_{q}(x)=f_{q}(x) \quad \forall x \in P,
$$

which is $B(q, q)$-invariant since $f$ is $\mathcal{B}_{G}$-equivariant. For the same reason,

$$
g_{r}(x)=f_{r}(x) \quad \forall x \in P, r \in Q,
$$

where $g_{r}$ is defined as in Lemma 4.5. Recall that $g_{s}(x)=0$ for all $s \notin Q$. Repeating this

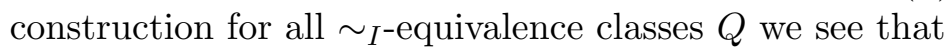

$$
\mathcal{F}_{G}^{P}=\sum \mathcal{F}_{G}^{P}(Q)
$$

However, the definition of $\mathcal{F}_{G}^{P}(Q)$ shows that

$$
\mathcal{F}_{G}^{P}(Q) \cap \sum_{R \neq Q} \mathcal{F}_{G}^{P}(R)=\{0\}
$$

(where $R$ ranges over $\sim_{I}$-equivalence classes other than $Q$ ), so the sum is direct.

5. Patterns of synchrony: Example. There are many kinds of synchrony in coupled cell systems: for surveys see Boccaletti, Pecora, and Pelaez [2] and Wang [14]. Most notions of synchrony depend on specific dynamics of cells and couplings. Some notions are model-independent; that is, they are valid for any vector field consistent with the given cell architecture. We believe that it is useful to distinguish model-independent properties from model-dependent ones, because this separates the effect of the general architecture of the system from that of the specific model equations employed, which clarifies the role of the model and its parameters. 
We now approach the central issue of this paper: conditions under which certain cells in a coupled cell network can synchronize as a consequence of the network architecture. Because the theoretical issues are somewhat abstract, we first discuss a motivating example.

Example 5.1. Consider the ten-cell network $G_{1}$ of Figure 11. There are two cell types. Cells 0 and 1 have type $\bigcirc$, and cells $2,3,4,5,6,7,8,9$ have type $\square$. There are three arrow types $\longrightarrow, \Longrightarrow,--\rightarrow$. The shading on the nodes divides $\mathcal{C}$ into three classes:

$\{0,1\},\{2,3,6,8\},\{4,5,7,9\}$.

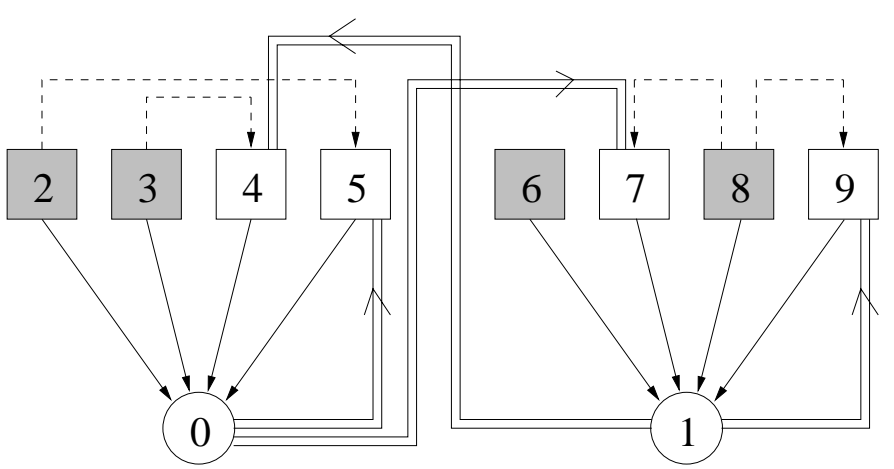

Figure 11. A 10-cell system. The shading indicates a possible pattern of synchronous cells.

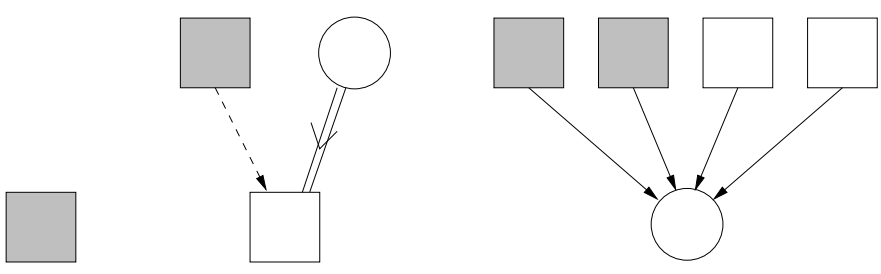

Figure 12. Input types for the 10-cell system, including shading.

There are three distinct input types, illustrated in Figure 12. Cells 2, 3, 6, 8 have no inputs except themselves. Cells 4, 5, 7, 9 have two inputs: one is of type $\bigcirc$, and the other is of type $\square$ and in the class $\{2,3,6,8\}$. Cells 0,1 have four inputs, all of type $\square$; of these, two are in the class $\{2,3,6,8\}$, and the other two are in the class $\{4,5,7,9\}$.

(We have deliberately included some cells without inputs - in this case, cells 2, 3, 6, and 8 - to make it clear that such cells can be considered synchronous in our formalism. Of course such a form of synchrony is dynamically unstable, but in this paper we are studying existence, not stability, and we do not wish to rule out unstable synchrony because it still corresponds to an invariant subspace for the dynamics. It is easy to modify this example to add further connections that provide inputs to these cells: for example, each of cells $2,3,6$, and 8 can receive one input from any of the cells $4,5,7$, and 9.) 
With appropriate choice of phase spaces, a vector field $f \in \mathcal{F}_{G_{1}}^{P}$ takes the form

$$
\begin{array}{ll}
f_{0}=A\left(x_{0}, \overline{x_{2}, x_{3}, x_{4}, x_{5}}\right), & f_{5}=C\left(x_{5}, x_{0}, x_{2}\right), \\
f_{1}=A\left(x_{1}, \overline{x_{6}, x_{7}, x_{8}, x_{9}}\right), & f_{6}=B\left(x_{6}\right), \\
f_{2}=B\left(x_{2}\right), & f_{7}=C\left(x_{7}, x_{0}, x_{8}\right), \\
f_{3}=B\left(x_{3}\right), & f_{8}=B\left(x_{8}\right), \\
f_{4}=C\left(x_{4}, x_{1}, x_{3}\right), & f_{9}=C\left(x_{9}, x_{1}, x_{8}\right) .
\end{array}
$$

Consider the space

$$
Y=\{(u, u, v, v, w, w, v, w, v, w)\}
$$

determined by making entries constant on the classes (5.1). On $Y$ the vector field $f$ restricts to

$$
\begin{array}{ll}
g_{0}=A(u, \overline{v, v, w, w}), & g_{5}=C(w, u, v), \\
g_{1}=A(u, \overline{v, w, v, w}), & g_{6}=B(v), \\
g_{2}=B(v), & g_{7}=C(w, u, v), \\
g_{3}=B(v), & g_{8}=B(v), \\
g_{4}=C(w, u, v), & g_{9}=C(w, u, v) .
\end{array}
$$

By symmetry $g_{0}, g_{1}$ are identical. Bearing this in mind, we see that $Y$ is flow-invariant for $f$. Identifying elements of $Y$ with triples $(u, v, w)$, we obtain an induced vector field $\bar{f}$ of the form

$$
\begin{aligned}
& \bar{f}_{0}=A^{\prime}(u, \bar{v}, w), \\
& \bar{f}_{1}=B(v), \\
& \bar{f}_{3}=C(w, u, v),
\end{aligned}
$$

where

$$
A^{\prime}(u, \overline{v, w})=A(u, \overline{v, v, w, w}) .
$$

This is the class of admissible vector fields for the simpler coupled cell network $G_{2}$ shown in Figure 13. Here cells $v, w$ have the same type, but we have shaded cell $v$ to show which equivalence class it corresponds to.

The coupled cell network $G_{2}$ is an example of a quotient network. What structure in $G_{1}$ makes $Y$ flow-invariant for all $f \in \mathcal{F}_{G_{1}}^{P}$ and permits this reduction to $G_{2}$ on $Y$ ? The key feature is how the three classes (5.1) relate to input isomorphisms. In section 6 we develop the theory of flow-invariant subspaces, and in section 8 we develop the general theory of such reductions.

6. Patterns of synchrony: Theory. We now isolate the abstract features of Example 5.1 that make $Y$ flow-invariant. The classes (5.1) can be represented as the equivalence classes corresponding to an equivalence relation. The properties of this equivalence relation, relative to the symmetry groupoid of the network, turn out to control the existence of the flow-invariant subspace $Y$ and the quotient network $G_{2}$. We begin by considering the equivalence relation. 


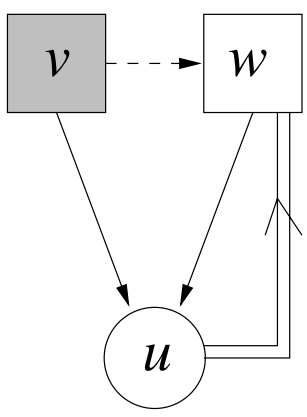

Figure 13. Quotient network $G_{2}$ of the 10 -cell system $G_{1}$. Shading relates cells to those in $G_{1}$.

Let $G=\left(\mathcal{C}, \mathcal{E}, \sim_{C}, \sim_{E}\right)$ be a coupled cell network. Choose a total phase space $P$, and let $\bowtie$ be an equivalence relation on $\mathcal{C}$, partitioning the cells into equivalence classes. We assume that $\bowtie$ is a refinement of $\sim_{\mathcal{C}}$; that is, if $c \bowtie d$, then $c$ and $d$ have the same cell labels. It follows that the polydiagonal subspace

$$
\Delta_{\bowtie}=\left\{x \in P: x_{c}=x_{d} \text { whenever } c \bowtie d \quad \forall c, d \in \mathcal{C}\right\}
$$

is well defined since $x_{c}$ and $x_{d}$ lie in the same space $P_{c}=P_{d}$. The polydiagonal $\Delta_{\bowtie}$ is a linear subspace of $P$.

For instance, in Example 5.1 we can define $\bowtie$ to have equivalence classes (5.1), in which case $\Delta_{\bowtie}=Y$.

Definition 6.1. A trajectory $x(t)$ of $f \in \mathcal{F}_{G}^{P}$ is $\bowtie$-polysynchronous if its components are constant on $\bowtie$-equivalence classes. That is,

$$
c \bowtie d \Longrightarrow x_{c}(t)=x_{d}(t) \quad \forall t \in \mathbf{R}
$$

or $x(t) \in \Delta_{\bowtie}$ for all $t \in \mathbf{R}$.

Polysynchronous states are patterns of synchrony. Trivially, any trajectory is polysynchronous with respect to the relation of equality (which partitions $\mathcal{C}$ into its individual cells). Only nontrivial polysynchrony is interesting.

\section{Robust polysynchrony.}

Definition 6.2. Let $\bowtie$ be an equivalence relation on $\mathcal{C}$. Then $\bowtie$ is robustly polysynchronous if $\Delta_{\bowtie}$ is invariant under every vector field $f \in \mathcal{F}_{G}^{P}$. That is,

$$
f\left(\Delta_{\bowtie}\right) \subseteq \Delta_{\bowtie} \quad \forall f \in \mathcal{F}_{G}^{P} .
$$

Equivalently, if $x(t)$ is a trajectory of any $f \in \mathcal{F}_{G}^{P}$, with initial condition $x(0) \in \Delta_{\bowtie}$, then $x(t) \in \Delta_{\bowtie}$ for all $t \in \mathbf{R}$.

We now find necessary and sufficient conditions on $\bowtie$ to ensure that $\bowtie$ is robustly polysynchronous. We begin by showing that robust polysynchrony can occur only between cells that have isomorphic input sets. This is intuitively clear because these are the only cells that involve the "same" function in the corresponding components of admissible vector fields, and the proof bears out this intuition. 
Lemma 6.3. If $\bowtie$ is robustly polysynchronous, then $\bowtie$ refines $\sim_{I}$. That is, for all $c, d \in \mathcal{C}$

$$
c \bowtie d \Longrightarrow c \sim_{I} d .
$$

Proof. By the discussion immediately preceding Lemma 3.8 we need to show that if $c \bowtie d$, then $c$ and $d$ are in the same connected component $Q$ of $\mathcal{B}_{G}$. Suppose they are not; then we will show that $\Delta_{\bowtie}$ is not flow-invariant. Choose $x(0) \in \Delta_{\bowtie}$ so that $x_{c}(0)=x_{d}(0) \neq 0$, and choose $f \in \mathcal{F}_{G}^{P}(Q)$, where $d \notin Q$. Let $x(t)$ be the solution to the differential equation $f$. Since $f \in \mathcal{F}_{G}^{P}(Q)$ implies that $f_{d}$ vanishes, $x_{d}(t)=x_{d}(0)$ for all $t$. If we can choose $f$ so that $f_{c}(x(0)) \neq 0$, then $x_{c}(t) \neq x_{c}(0)$ for small $t$, so $\Delta_{\bowtie}$ is not flow-invariant, and we are finished.

It remains to choose such an $f$. By Lemma 4.5 we need only find a $B(c, c)$-invariant mapping $g_{c}: P_{I(c)} \rightarrow P_{c}$ such that $g_{c}(x(0)) \neq 0$, since such an invariant mapping extends to a vector field in $\mathcal{F}_{G}^{P}(Q)$. For example, we may take $g_{c}(x)=x_{c}(0) \neq 0$, which is $B(c, c)$-invariant.

Balanced equivalence relations. In order to motivate our characterization of robustly synchronous equivalence relations $\bowtie$, we repeat the analysis of Example 5.1 with a slightly different equivalence relation. Suppose that we partition the ten cells into the classes

$$
\{0,1\},\{2,3,8\},\{4,5,6,7,9\}
$$

so that the color of cell 6 is now gray instead of white, all other colors remaining as in Figure 11. Now the associated polydiagonal is

$$
Y^{\prime}=\{(u, u, v, v, w, w, w, w, v, w)\} .
$$

The general vector field (5.2) remains unchanged, but its restriction (5.3) to $Y$ changes in just one component: now

$$
g_{1}=A(u, \overline{w, w, v, w})
$$

This is no longer the same as $g_{0}$, so no reduction to the three-cell network is possible.

What is the source of this difference? The symmetry property of $A$ (that is, its $B(1,1)$ invariance) implies that the order of the $v$ 's and $w$ 's does not matter, but there are three occurrences of $w$ in $g_{1}$ and only two occurrences in $g_{0}$. Similarly there is one occurrence of $v$ in $g_{1}$, but there are two occurrences in $g_{0}$. This difference in "multiplicity" makes $g_{1}$ differ from $g_{0}$ and so destroys the possibility of $Y^{\prime}$ being an invariant subspace.

This and similar examples lead to the following concept.

Definition 6.4. An equivalence relation $\bowtie$ on $\mathcal{C}$ is balanced if for all $c, d \in \mathcal{C}$ with $c \bowtie d$ and $c \neq d$, there exists $\gamma \in B(c, d)$ such that $i \bowtie \gamma(i)$ for all $i \in I(c)$.

In particular, $B(c, d) \neq \emptyset$ implies $c \sim_{I} d$. Therefore, balanced equivalence relations refine $\sim_{I}$

The equivalence relation for Example 5.1 is balanced; the modified equivalence relation is not balanced. It turns out that this is the crucial distinction when it comes to constructing a quotient network; see Theorem 6.5 below. 
There is a relatively simple graphical way to test whether a given equivalence relation $\bowtie$ is balanced. Color the cells in a network so that two cells have the same color precisely when they are in the same $\bowtie$-equivalence class. Then $\bowtie$ is balanced if and only if every pair of $\bowtie$-equivalent cells is connected by a color preserving groupoid element.

For example, consider the seven-cell network in Figure 14. Let $\bowtie$ be the equivalence relation with equivalence classes

$$
\{1,4,7\}, \quad\{2,5\}, \quad\{3,6\},
$$

as indicated by the colors in Figure 14. Observe that the pink (light gray) cells have input sets "white to pink," the white cells have input sets "blue (dark gray) to white," and the blue cells have input sets "pink to blue." So $\bowtie$ is a balanced equivalence relation, since all cells in the same equivalence class have identically colored input sets.

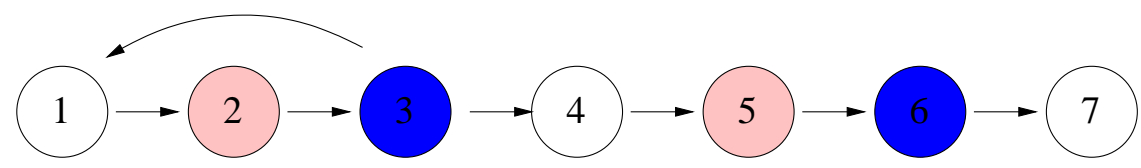

Figure 14. Seven-cell linear network with $\bowtie$-equivalence classes indicated by color.

The main theorem on polysynchrony. An examination of these examples leads to the following general result.

Theorem 6.5. Let $\bowtie$ be an equivalence relation on a coupled cell network. Then $\bowtie$ is robustly polysynchronous if and only if $\bowtie$ is balanced.

Proof. If $\bowtie$ is balanced, then Definition 6.4 implies that $\Delta_{\bowtie}$ is invariant under any admissible vector field; that is, $\bowtie$ is robustly polysynchronous. This is obvious in the "color" interpretation: as we have seen, $\bowtie$ is balanced if and only if every pair of cells of the same color are related by a color preserving input isomorphism. This implies that if $f \in \mathcal{F}_{G}^{P}$ and $c \bowtie d$, then $f_{c}(x)=f_{d}(x)$ for all $x \in \Delta_{\bowtie}$. That is, $\Delta_{\bowtie}$ is flow-invariant for $f$.

To prove the converse, suppose that $\bowtie$ is robustly polysynchronous. Then every $f \in \mathcal{F}_{G}^{P}$

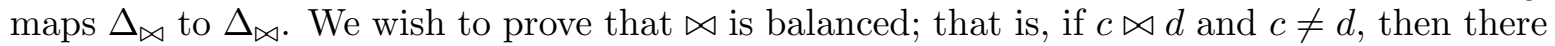
exists $\gamma \in B(c, d)$ such that $i \bowtie \gamma(i)$ for all $i \in I(c)$. Since $\bowtie$ refines $\sim_{I}$ (Lemma 6.3), the set $B(c, d)$ is nonempty.

Define $K_{0}, \ldots, K_{r}$ as in (3.4) so that there is a partition

$$
I(c)=K_{0} \dot{\cup} \cdots \dot{U} K_{r},
$$

where $i, i^{\prime}$ belong to the same $K_{s}$ if and only if $(i, c) \sim_{E}\left(i^{\prime}, c\right)$. Similarly, there is a partition

$$
I(d)=L_{0} \dot{\cup} \cdots \dot{U} L_{r}
$$

with the corresponding property. We may choose the numbering so that

$$
i \in K_{s}, j \in L_{s} \Longrightarrow(i, c) \sim_{E}(j, d), \quad 1 \leq s \leq r .
$$

As before, we may take $K_{0}=\{c\}, L_{0}=\{d\}$. (Because $c \sim_{I} d$, the sets $K_{s}$ and $L_{s}$ have the same cardinality for $0 \leq s \leq r$, and the same $r$ occurs for $I(c)$ and $I(d)$.) 
Suppose that we can prove that for any $s$ with $0 \leq s \leq r$ and any $\bowtie$-equivalence class $U \subseteq \mathcal{C}$,

$$
\left|U \cap K_{s}\right|=\left|U \cap L_{s}\right|
$$

Then we can define a bijection $\gamma: I(c) \rightarrow I(d)$ such that

$$
\gamma\left(U \cap K_{s}\right)=U \cap L_{s} \quad(0 \leq s \leq r)
$$

for all $U$. By (6.1), $\gamma \in B(c, d)$. Moreover, (6.3) implies that $\gamma(i) \bowtie i$ for all $i \in I(c)$. For we may take $U$ such that $i \in U$, and then $\gamma(i) \in U$ as well.

Thus it remains to prove the cardinality condition (6.2). To do so, we introduce a $\mathcal{B}_{G^{-}}$ equivariant map $h$, which depends on $s$, and apply it to an element $y \in \Delta_{\bowtie}$ that depends on $U$, as follows.

Let $M: P_{i} \rightarrow P_{c}$ be a nonzero linear map, where $i \in K_{s}$. Let $h_{c}: P_{I(c)} \rightarrow P_{c}$ be defined by

$$
h_{c}(x)=M\left(\sum_{i \in K_{s}} x_{i}\right),
$$

which is $B(c, c)$-invariant, since $K_{s}$ is a $B(c, c)$-orbit. By Lemma 4.5 we may then define, for all other $c^{\prime} \in \mathcal{C}$,

$$
h_{c^{\prime}}(x)=h_{c}\left(\beta^{*}(x)\right)=\sum_{j \in \beta\left(K_{s}\right)} M\left(x_{j}\right)
$$

where $\beta$ is some (hence any) element of $B\left(c, c^{\prime}\right)$ and $c^{\prime} \sim_{I} c$, and

$$
h_{c^{\prime}}(x)=0
$$

otherwise. Moreover, the resulting $h$ is $\mathcal{B}_{G}$-equivariant. Since $\bowtie$ is polysynchronous, $h$ maps $\Delta_{\bowtie}$ to itself.

Next, define $y \in P$ by

$$
y_{j}= \begin{cases}v, & j \in U \\ 0, & j \notin U\end{cases}
$$

for some fixed $v \in P_{a}$ for which $M(v) \neq 0$, where $a \in U \cap K_{s}$.

We are assuming that $c \sim_{I} d$ so that $B(c, d) \neq \emptyset$. Let $\beta \in B(c, d)$, which implies that $\beta\left(K_{s}\right)=L_{s}$. Clearly

$$
h_{d}(x)=\sum_{j \in L_{s}} M\left(x_{j}\right) \quad \forall x \in \Delta_{\bowtie} .
$$

Since $h$ preserves $\Delta_{\bowtie}$,

$$
h_{c}(x)=h_{d}(x) \quad \forall x \in \Delta_{\bowtie} .
$$


Apply (6.5),(6.6) to $y$ :

$$
\begin{aligned}
& h_{c}(y)=\left|U \cap K_{s}\right| M(v), \\
& h_{d}(y)=\left|U \cap L_{s}\right| M(v) .
\end{aligned}
$$

By (6.6), since $M(v) \neq 0$, we deduce that

$$
\left|U \cap K_{s}\right|=\left|U \cap L_{s}\right|
$$

for all $U$ and all $0 \leq s \leq r$. However, this is (6.2), so $\bowtie$ is balanced.

7. Dynamics on polysynchronous subspaces. As illustrated in Example 5.1 the restriction of a coupled cell vector field to a polysynchronous subspace has itself a special structure. The restriction is an admissible vector field for an associated "quotient" coupled cell network. In this section we construct the quotient network for a given polysynchronous subspace and illustrate some of the implications for the dynamics of the restriction. We begin with an example.

Example 7.1. Consider the five-cell network illustrated in Figure 15 (left). All cells are cellequivalent, so a phase space for this network has the form $P=\left(\mathbf{R}^{k}\right)^{5}$ for some $k$. Since all cells are also input-equivalent (that is, have isomorphic input sets) the diagonal $(x, x, x, x, x)$ is polysynchronous. Recall that a network is homogeneous when all of its cells are input isomorphic.
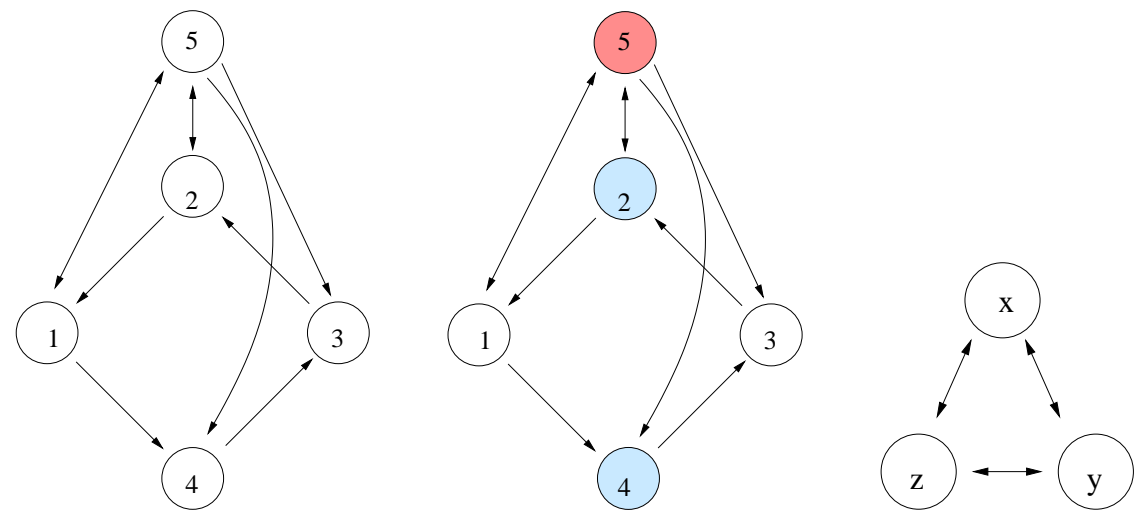

Figure 15. A homogeneous five-cell network with a balanced relation leading to a quotient three-cell bidirectional ring.

There is, however, a more interesting $3 k$-dimensional polysynchronous subspace $\Delta_{\bowtie}$ associated to the balanced relation illustrated in Figure 15 (center). That subspace is

$$
\Delta_{\bowtie}=\left\{(x, y, x, y, z): x, y, z \in \mathbf{R}^{k}\right\} .
$$

Next we discuss the structure of the restriction of an admissible coupled cell vector field 


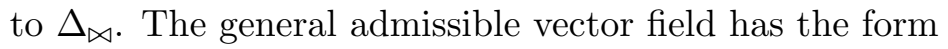

$$
\begin{aligned}
& \dot{x}_{1}=f\left(x_{1}, \overline{x_{2}, x_{5}}\right), \\
& \dot{x}_{2}=f\left(x_{2}, \overline{x_{3}, x_{5}}\right), \\
& \dot{x}_{3}=f\left(x_{3}, \overline{x_{4}, x_{5}}\right), \\
& \dot{x}_{4}=f\left(x_{4}, \overline{x_{1}, x_{5}}\right), \\
& \dot{x}_{5}=f\left(x_{5}, \overline{x_{1}, x_{2}}\right),
\end{aligned}
$$

where $f:\left(\mathbf{R}^{k}\right)^{3} \rightarrow \mathbf{R}^{k}$ is symmetric in the last two arguments. The restriction of (7.1) to $\Delta_{\bowtie}$ has the form

$$
\begin{aligned}
\dot{x} & =f(x, \overline{y, z}), \\
\dot{y} & =f(y, \overline{z, x}), \\
\dot{z} & =f(z, \overline{x, y}) .
\end{aligned}
$$

Observe that (7.2) is the general vector field associated to the three-cell bidirectional ring illustrated in Figure 15 (right). We will show that there is a general construction that leads to this three-cell quotient, but first we discuss some implications for the dynamics of the five-cell system.

Observe also that the restriction (7.2) has $\mathbf{D}_{3}$ symmetry and is, in fact, the general $\mathbf{D}_{3^{-}}$ equivariant vector field on $\left(\mathbf{R}^{k}\right)^{3}$. So it is possible for a quotient network to have symmetry even when the original network has none. It is known that when $k \geq 2$, such vector fields can support discrete rotating waves and solutions where two cells are out of phase, while the third cell has twice the frequency of the other two [9, 7]. These solutions are also solutions to the original five-cell system. Typical simulations are shown in Figure 16. The middle and right simulations are obtained just by changing initial conditions.
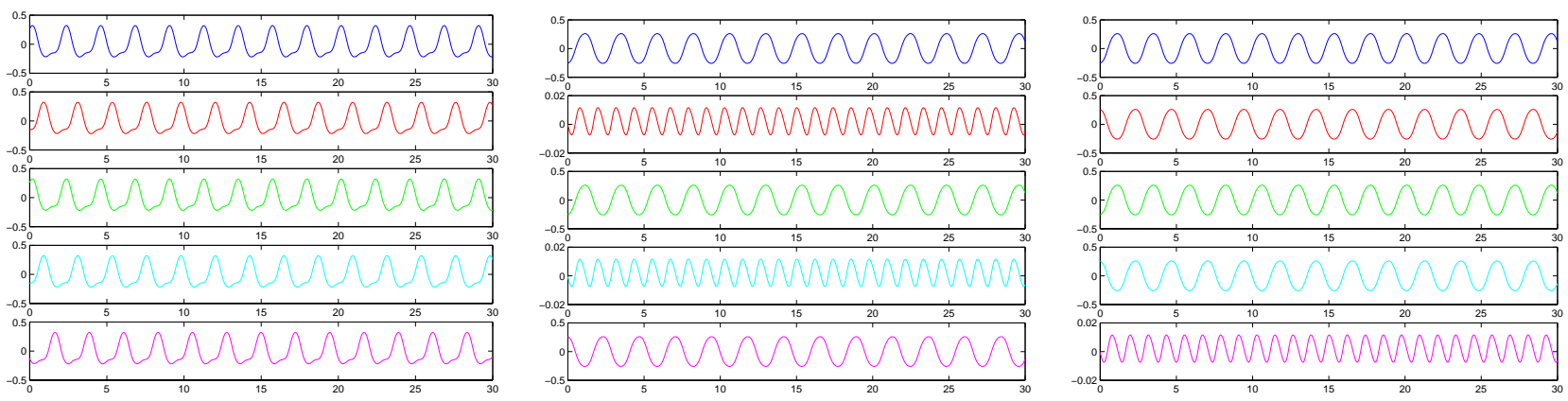

Figure 16. Simulations in homogeneous five-cell network in Figure 15. (Left) rotating wave; (middle) double frequency in cells 2 and 4; (right) double frequency in cell 5.

It is also possible for the restricted system to exhibit symmetric chaos, as illustrated in the five-cell simulations in Figures 17 and 18.

Perhaps the simplest example of a network that has no symmetry but does have a quotient network with symmetry is the three-cell network in Figure 19. This is the same as the "masterslave" network of Pecora and Carroll [12]. This network is part of the same family of networks as the seven-cell network described in section 1 (Figure 6) and again in section 6 (Figure 14). 

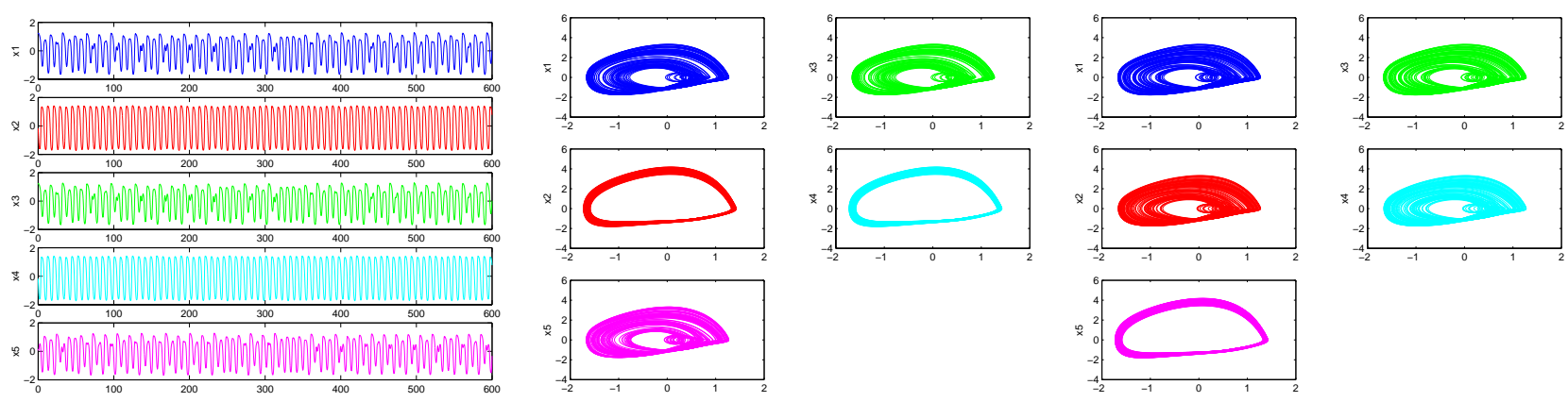

Figure 17. Simulations in homogeneous five-cell network in Figure 15. (Left) time series for chaotic attractor with $\mathbf{Z}_{2}$ symmetry; (middle) phase plane with cells 1,3,5 and cells 2, 4 exhibiting symmetry on average; (right) double frequency in cell 5.
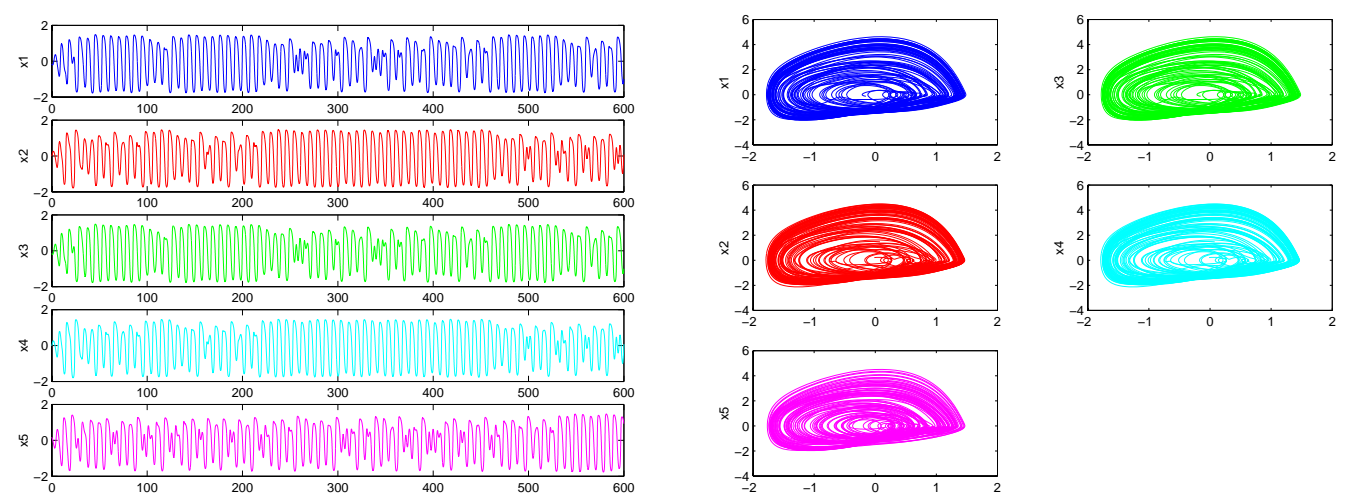

Figure 18. Simulations in homogeneous five-cell network in Figure 15. (Left) time series for chaotic attractor with $\mathbf{Z}_{3}$ symmetry; (right) phase planes with all cells exhibiting symmetry on average.

Construction of the natural quotient network. Let $\bowtie$ be a balanced equivalence relation on a coupled cell network $G=\left(\mathcal{C}, \mathcal{E}, \sim_{C}, \sim_{E}\right)$. In a series of steps we construct the quotient network $G_{\bowtie}$ corresponding to the polysynchronous subspace $\Delta_{\bowtie}$. To do this we need to define the cells and edges of the quotient network and the equivalence relations on them; that is, we must define $\mathcal{C}_{\bowtie}, \sim_{C_{\bowtie}}, \mathcal{E}_{\bowtie}, \sim_{E_{\bowtie}}$. Most steps are straightforward, but those related to edge-equivalence are more complicated.

(A) Let $\bar{c}$ denote the $\bowtie$-equivalence class of $c \in \mathcal{C}$. The cells in $\mathcal{C}_{\bowtie}$ are the $\bowtie$-equivalence classes in $\mathcal{C}$; that is,

$$
\mathcal{C}_{\bowtie}=\{\bar{c}: c \in \mathcal{C}\}
$$

Thus we obtain $\mathcal{C}_{\bowtie}$ by forming the quotient of $\mathcal{C}$ by $\bowtie$; that is, $\mathcal{C}_{\bowtie}=\mathcal{C} / \bowtie$.

(B) Define

$$
\bar{c} \sim_{C_{\triangleright}} \bar{d} \Longleftrightarrow c \sim_{C} d
$$

This is well defined since $\bowtie$ refines $\sim_{C}$. 


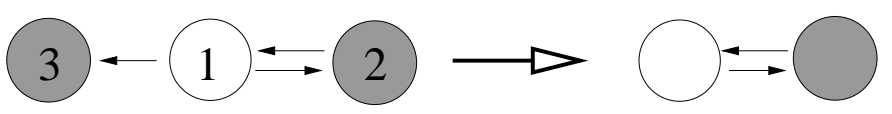

Figure 19. A three-cell network with no symmetry having a quotient two-cell network with $\mathbf{Z}_{2}$ symmetry.

(C) The edges in the quotient network are the projection of edges in the original network that do not link distinct but $\bowtie$-equivalent cells. That is,

$$
\mathcal{E}_{\bowtie}=\{(\bar{i}, \bar{c}):(i, c) \in \mathcal{E}, i \not \downarrow c\} \cup\{(\bar{c}, \bar{c}): c \in \mathcal{C}\} .
$$

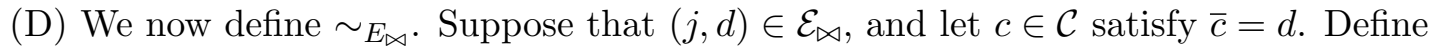

$$
\Omega_{c}(j)=\{i \in I(c): \bar{i}=j\} .
$$

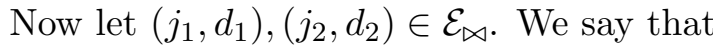

$$
\left(j_{1}, d_{1}\right) \sim_{E_{\bowtie}}\left(j_{2}, d_{2}\right)
$$

if and only if for some $c_{1}, c_{2} \in \mathcal{C}$ with $\overline{c_{1}}=d_{1}, \overline{c_{2}}=d_{2}$ there exists $\gamma \in B\left(c_{1}, c_{2}\right)$ such that

$$
\gamma\left(\Omega_{c_{1}}\left(j_{1}\right)\right)=\Omega_{c_{2}}\left(j_{2}\right)
$$

Remark 7.2. We interrupt our discussion to provide a word picture of the construction of edge-equivalence $\sim_{E_{\bowtie}}$. Suppose that the cells of $\mathcal{C}$ are colored by $\bowtie$-equivalence classes, as discussed previously. So every cell in $\mathcal{C}_{\bowtie}$ can be identified with a unique color. The set $\Omega_{c}(j)$ consists of those cells in the input set $I(c)$ having color $j$. The edges $\left(j_{1}, d_{1}\right)$ and $\left(j_{2}, d_{2}\right)$ are $\sim_{E_{\bowtie}}$-equivalent if there is an input equivalence of $I\left(c_{1}\right)$ to $I\left(c_{2}\right)$ that maps cells of color $j_{1}$ to cells of color $j_{2}$. In particular, the number of cells in $I\left(c_{1}\right)$ of color $j_{1}$ must equal the number of cells in $I\left(c_{2}\right)$ of color $j_{2}$.

This completes the construction of $G_{\bowtie}=\left(\mathcal{C}_{\bowtie}, \mathcal{E}_{\bowtie}, \sim_{C_{\bowtie}}, \sim_{E_{\bowtie}}\right)$, except for one final technical remark. As stated, the definition of $\sim_{E_{\bowtie}}$ appears to depend on the choice of $c_{1}, c_{2}$ in (D). In fact, it does not.

Lemma 7.3. Suppose that $\bowtie$ is balanced. Let $c_{1}, c_{2}, c_{1}^{\prime}, c_{2}^{\prime} \in \mathcal{C}$, where $c_{1} \bowtie c_{1}^{\prime}$ and $c_{2} \bowtie c_{2}^{\prime}$. Let $j_{1}, j_{2} \in \mathcal{C}_{\bowtie}$. Suppose that there exists $\beta \in B\left(c_{1}, c_{2}\right)$ such that

$$
\beta\left(\Omega_{c_{1}}\left(j_{1}\right)\right)=\Omega_{c_{2}}\left(j_{2}\right) .
$$

Then there exists $\beta^{\prime} \in B\left(c_{1}^{\prime}, c_{2}^{\prime}\right)$ such that

$$
\beta^{\prime}\left(\Omega_{c_{1}^{\prime}}\left(j_{1}\right)\right)=\Omega_{c_{2}^{\prime}}\left(j_{2}\right)
$$

Proof. Since $\bowtie$ is balanced, there exists (for $k=1,2)$ an element $\gamma_{k} \in B\left(c_{k}, c_{k}^{\prime}\right)$ such that $\gamma_{k}(i) \bowtie i$ for all $i \in I\left(c_{k}\right)$. Therefore,

$$
\gamma_{k}\left(\Omega_{c_{k}}\left(j_{k}\right)\right)=\Omega_{c_{k}^{\prime}}\left(j_{k}\right)
$$


Clearly $\beta^{\prime}=\gamma_{2} \beta \gamma_{1}^{-1}$ is an input isomorphism and by construction $\beta^{\prime}\left(\Omega_{c_{1}^{\prime}}\left(j_{1}\right)\right)=\Omega_{c_{2}^{\prime}}\left(j_{2}\right)$.

Lemma 7.3 implies that if (D) holds for some choice of $c_{1}, c_{2}$ satisfying the required conditions, then it holds for any choice of $c_{1}, c_{2}$.

Finally, we show that $G_{\bowtie}$ is a coupled cell network. To do so, we must verify the compatibility conditions in Definition 2.1(e,f).

(E) If $\left(j_{1}, d_{1}\right) \sim_{E_{\bowtie}}\left(j_{2}, d_{2}\right)$, then $j_{1} \sim_{C_{\bowtie}} j_{2}$ and $d_{1} \sim_{C_{\bowtie}} d_{2}$.

Choose $c_{1}, c_{2} \in \mathcal{C}$ such that $\overline{c_{1}}=d_{1}$ and $\overline{c_{2}}=d_{2}$. The definition of $\sim_{E_{\bowtie}}$ implies there exists $\gamma \in B\left(c_{1}, c_{2}\right)$ such that $\gamma\left(\Omega_{c_{1}}\left(j_{1}\right)\right)=\Omega_{c_{2}}\left(j_{2}\right)$. Since $\gamma$ is an input isomorphism, it preserves cell type, so $c_{1} \sim_{C} c_{2}$. However, now the definition of $\sim_{C_{\bowtie}}$ shows that $d_{1} \sim_{C_{\bowtie}} d_{2}$. Next choose any $i \in \Omega_{c_{1}}\left(j_{1}\right)$. Then $\gamma(i) \in \Omega_{c_{2}}\left(j_{2}\right)$, and $i \sim_{C} \gamma(i)$. Therefore, $j_{1} \sim_{C_{\triangleright}} j_{2}$.

(F) Internal edges are never equivalent to noninternal ones; that is,

$$
\left(j_{1}, j_{1}\right) \sim_{E_{\bowtie}}\left(j_{2}, d_{2}\right) \Longleftrightarrow j_{2}=d_{2} \text { and } j_{2} \sim_{C_{\bowtie}} j_{1}
$$

for all $j_{1}, j_{2}, d_{2} \in \mathcal{C}_{\bowtie \text {. }}$

We prove $\Longrightarrow$. Assume that $\left(j_{1}, j_{1}\right) \sim_{E_{\bowtie}}\left(j_{2}, d_{2}\right)$ and choose $c_{1}, c_{2} \in \mathcal{C}$ such that $\overline{c_{1}}=$ $j_{1}$ and $\overline{c_{2}}=d_{2}$. The definition of $\sim_{E_{\bowtie}}$ implies that there exists $\gamma \in B\left(c_{1}, c_{2}\right)$ satisfying (7.4): $\gamma \Omega_{c_{1}}\left(j_{1}\right)=\gamma \Omega_{c_{2}}\left(j_{2}\right)$. Suppose that $\Omega_{c_{1}}\left(j_{1}\right)=\left\{c_{1}, i_{1}, \ldots, i_{p}\right\}$. Then $\Omega_{c_{2}}\left(j_{2}\right)=$ $\left\{\gamma\left(c_{1}\right), \gamma\left(i_{1}\right), \ldots, \gamma\left(i_{p}\right)\right\}=\left\{c_{2}, \gamma\left(i_{1}\right), \ldots, \gamma\left(i_{p}\right)\right\}$ and $d_{2}=\bar{c}_{2}=\overline{\gamma\left(i_{1}\right)}=\cdots=\overline{\gamma\left(i_{p}\right)}=j_{2}$ by definition of $\Omega_{c_{2}}\left(j_{2}\right)$. Thus $j_{2}=d_{2}$. As $j_{2}=\bar{c}_{2}, j_{1}=\bar{c}_{1}$, and $c_{1} \sim_{c} c_{2}$ (since $B\left(c_{1}, c_{2}\right) \neq \emptyset$ ), then $j_{1} \sim_{C_{\bowtie}} j_{2}$.

The converse is obtained by direct calculation.

It remains to prove that the restriction of each $G$-admissible vector field to $\Delta_{\bowtie}$ is a $G_{\bowtie^{-}}$ admissible vector field. This result follows from Theorem 9.2, whose proof uses "quotient maps," which are introduced in section 8.

Remark 7.4 (on the symmetry groupoid of the natural quotient). It is reasonable to ask for a characterization of the symmetry groupoid of the natural quotient $G / \bowtie$ in terms of the symmetry groupoid of $G$ and its relation to $\bowtie$.

Define

$$
\begin{aligned}
& \Sigma^{\bowtie}(c, d)=\{\sigma \in B(c, d): \sigma(i) \bowtie i \quad \forall i \in I(c)\}, \\
& \mathrm{T}^{\bowtie}(c, d)=\{\tau \in B(c, d): i \bowtie j \Longleftrightarrow \tau(i) \bowtie \tau(j) \quad \forall i, j \in I(c)\} .
\end{aligned}
$$

Then define two subgroupoids of $\mathcal{B}_{G}$ by

$$
\begin{aligned}
\Sigma^{\bowtie} & =\bigcup_{c, d \in \mathcal{C}} \Sigma^{\bowtie}(c, d), \\
\mathrm{T}^{\bowtie} & =\bigcup_{c, d \in \mathcal{C}} \mathrm{T}^{\bowtie}(c, d) .
\end{aligned}
$$

It turns out that $\mathrm{T}^{\bowtie}$ consists precisely of the $\bowtie$-compatible elements of $\mathcal{B}_{G}$, a concept that we introduce later in Definition 8.6. It follows that $\mathcal{B}_{G / \bowtie}$ consists precisely of the bijections induced on $\mathcal{C} / \bowtie$ by the subgroupoid $\mathrm{T}^{\bowtie}$ of $\mathcal{B}_{G}$.

Moreover, the elements of $\Sigma^{\bowtie}$ act as the identity on $\mathcal{C} / \bowtie$. In fact, they form the isotropy subgroupoid of any generic element of the polydiagonal $\Delta_{\bowtie}$ (that is, an element $x \in \Delta_{\bowtie}$ such that $x_{i}=x_{j} \Leftrightarrow i \bowtie j$ ). By analogy with the group-symmetric case, we expect $\mathcal{B}_{G / \bowtie}$ to be 
equal to the quotient groupoid $\mathrm{T}^{\bowtie} / \Sigma^{\bowtie}$. Moreover, $\mathrm{T}^{\bowtie}$ ought to be the "normalizer groupoid" of $\Sigma^{\bowtie}$ in $\mathcal{B}_{G}$.

Dias and Stewart [5] prove the above statements. We omit the proofs here because they involve technicalities about quotient groupoids that would take us too far afield.

Remark 7.5 (on the lifting of $G_{\bowtie}$ admissible vector fields). In symmetric dynamics the issue of "hidden symmetry" arises. Here, the restriction of an equivariant vector field onto the fixed-point space of a subgroup $\Sigma$ is always equivariant under the normalizer of $\Sigma$, but sometimes it obeys extra constraints. See $[9,7]$. The next example shows that the same issue arises in the groupoid context. In particular, vector fields that are admissible with respect to the quotient network $G_{\bowtie}$ do not always lift to vector fields that are admissible with respect to the original coupled cell network $G$.

Consider the four-cell network in Figure 20 (left). The equivalence relation $\bowtie$ indicated by color is balanced, and consider the natural quotient three-cell network in Figure 20 (right).

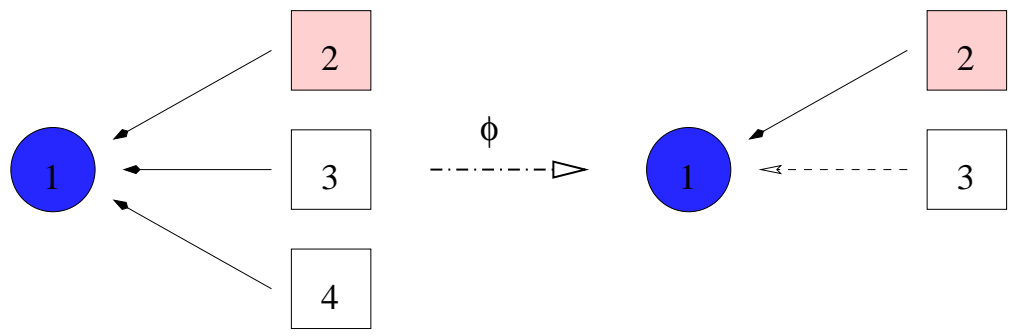

Figure 20. A four-cell example.

Admissible vector fields of the four-cell network have the form

$$
\begin{aligned}
& \dot{x}_{1}=A\left(x_{1}, \overline{x_{2}, x_{3}, x_{4}}\right) \\
& \dot{x}_{2}=B\left(x_{2}\right) \\
& \dot{x}_{3}=B\left(x_{3}\right) \\
& \dot{x}_{4}=B\left(x_{4}\right) .
\end{aligned}
$$

Admissible vector fields of the three-cell network have the form

$$
\begin{aligned}
& \dot{u}_{1}=f\left(u_{1}, u_{2}, u_{3}\right), \\
& \dot{u}_{2}=g\left(u_{2}\right), \\
& \dot{u}_{3}=g\left(u_{3}\right) .
\end{aligned}
$$

If we identify $\left(x_{1}, x_{2}, x_{3}, x_{4}\right)$ with $\left(u_{1}, u_{2}, u_{3}, u_{3}\right)$, we induce a vector field from (7.5), and we thereby obtain one of the form

$$
\begin{aligned}
& \dot{u}_{1}=A\left(u_{1}, \overline{u_{2}, u_{3}, u_{3}}\right) \\
& \dot{u}_{2}=B\left(u_{2}\right) \\
& \dot{u}_{3}=B\left(u_{3}\right)
\end{aligned}
$$

which is admissible by (7.6).

Note that not every $G_{\bowtie \text {-admissible vector field (7.6) }}$ can be extended to a $G$-admissible vector field. Compare the linear terms in $A$ (namely, $\alpha u_{1}+\beta\left(u_{2}+2 u_{3}\right)$ ) with the linear terms 
in $f$ in (7.6) (namely, $\alpha u_{1}+\beta u_{2}+\gamma u_{3}$ ). This is a groupoid analogue of hidden symmetry and raises similar issues. Dias and Stewart [5] give a complete groupoid-theoretic characterization

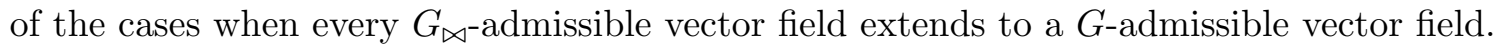

8. Quotient maps. In this section we give a formal definition of a quotient map $\phi: G_{1} \rightarrow$ $G_{2}$, where $G_{1}$ and $G_{2}$ are coupled cell networks. The definition is purely graph-theoretic.

Definition 8.1. Let $G_{i}=\left(\mathcal{C}_{i}, \mathcal{E}_{i}, \sim_{C_{i}}, \sim_{E_{i}}\right)$ be coupled cell networks. The map

$$
\phi: \mathcal{C}_{1} \rightarrow \mathcal{C}_{2}
$$

is a quotient map if the following hold:

(a) Cells lift: $\phi$ is surjective.

(b) Input arrows lift: If $(i, c) \in \mathcal{E}_{1}$, then $(\phi(i), \phi(c)) \in \mathcal{E}_{2}$. Conversely, if $(j, d) \in \mathcal{E}_{2}$ and $c \in \mathcal{C}_{1}$ such that $\phi(c)=d$, then there exists $i \in \mathcal{C}_{1}$ such that $\phi(i)=j$ and $(i, c) \in \mathcal{E}_{1}$.

(c) Input isomorphisms lift: Let $d, d^{\prime} \in \mathcal{C}_{2}$ and $\beta_{2} \in B\left(d, d^{\prime}\right)$. Choose $c, c^{\prime} \in \mathcal{C}_{1}$ such that $\phi(c)=d$ and $\phi\left(c^{\prime}\right)=d^{\prime}$. Then there exists $\beta_{1} \in B\left(c, c^{\prime}\right)$ such that

$$
\beta_{2}(\phi(i))=\phi\left(\beta_{1}(i)\right)
$$

for all $i \in I(c)$.

There are several observations that follow directly from the definition of a quotient map $\phi: \mathcal{C}_{1} \rightarrow \mathcal{C}_{2}$. Define the equivalence relation $\bowtie_{\phi}$ on $\mathcal{C}_{1}$ by

$$
c \bowtie_{\phi} c^{\prime} \Longleftrightarrow \phi(c)=\phi\left(c^{\prime}\right)
$$

That is, any two cells in $\mathcal{C}_{1}$ that project by $\phi$ onto the same cell in $\mathcal{C}_{2}$ have the same color (that is, are $\bowtie_{\phi}$-equivalent).

Lemma 8.2. Let $\phi: \mathcal{C}_{1} \rightarrow \mathcal{C}_{2}$ be a quotient map. Then the following hold:

(a) If $\phi(c)=d$, then

$$
\phi(I(c))=I(d)
$$

(b) For every $c, c^{\prime} \in \mathcal{C}_{1}$ such that $\phi(c)=\phi\left(c^{\prime}\right)$ there is an input isomorphism $\beta \in B\left(c, c^{\prime}\right)$ such that

$$
\phi(i)=\phi(\beta(i))
$$

for all $i \in I(c)$.

(c) The equivalence relation $\bowtie_{\phi}$ is balanced.

Proof. Part (a) follows directly from Definition 8.1 (b). Part (b) follows from Definition 8.1 (c) by setting $d=d^{\prime}$ and $\beta_{2}=$ id on $I(d)$. The existence of $\beta \in B\left(c, c^{\prime}\right)$ in Part (b) implies that $c \sim_{I_{1}} c^{\prime}$ and hence $c \sim_{C_{1}} c^{\prime}$. Using (8.2), identity (8.4) is equivalent to $i \bowtie_{\phi} \beta(i)$ for all $i \in I(c)$, which is the definition of "balanced" in Definition 6.4. Thus Part (c) holds. 


\section{Quotient networks are examples of quotient maps.}

Theorem 8.3. Assume that $\bowtie$ is a balanced equivalence relation on $\mathcal{C}$, and let $\mathcal{C}_{\triangleright}$ be the natural coupled cell network whose cells are the equivalence classes of $\bowtie$. Let $\bar{c}$ denote the $\bowtie$-equivalence class of the cell $c \in \mathcal{C}$. Then the map $\phi: \mathcal{C} \rightarrow \mathcal{C}_{\bowtie}$ defined by $c \mapsto \bar{c}$ is a quotient map.

Proof. We verify that $\phi: \mathcal{C} \rightarrow \mathcal{C}_{\bowtie}$ is a quotient map; that is, we verify Definition 8.1 (a) $-(\mathrm{c})$.

(a) Cells lift since $\phi$ is onto by construction.

(b) Input arrows lift by definition. See part $(\mathrm{C})$ in the construction of the network $\mathcal{C}_{\bowtie \text {. }}$

(c) We show that input isomorphisms lift. Recall that Definition 8.1 (c) states the following: For every $d, d^{\prime} \in \mathcal{C}_{\bowtie}, c, c^{\prime} \in \mathcal{C}$ such that $\bar{c}=d, \overline{c^{\prime}}=d^{\prime}$, and $\beta_{2} \in B\left(d, d^{\prime}\right)$, there exists $\beta_{1} \in B\left(c, c^{\prime}\right)$ such that $\beta_{2}(\bar{i})=\overline{\beta_{1}(i)}$ for all $i \in I(c)$. So we must construct the input isomorphism $\beta_{1}: I(c) \rightarrow I\left(c^{\prime}\right)$.

We first show that input sets lift; that is, $\overline{I(c)}=I(\bar{c})$ for each $c \in \mathcal{C}$. Suppose that $j \in I(\bar{c})$. Since input arrows lift, there exist $i^{\prime}, c^{\prime} \in \mathcal{C}$ such that $\overline{i^{\prime}}=j, \overline{c^{\prime}}=\bar{c}$, and $\left(i^{\prime}, c^{\prime}\right) \in \mathcal{E}$. By construction of $\mathcal{C}_{\bowtie}, c^{\prime}$ and $c$ are $\bowtie$-equivalent. Since $\bowtie$ is balanced, there exists $\gamma \in B\left(c^{\prime}, c\right)$ for which $i=\gamma\left(i^{\prime}\right) \bowtie i^{\prime}$. It follows that $\gamma\left(i^{\prime}, c^{\prime}\right)=(i, c) \in \mathcal{E}$ and that $\bar{i}=j$. Thus each input arrow in $I(\bar{c})$ lifts to an input arrow in $I(c)$.

Next we show that $\beta_{1}$ exists. The set $\Omega_{c}(j)$, defined in (7.3), consists of cells in $I(c)$ that

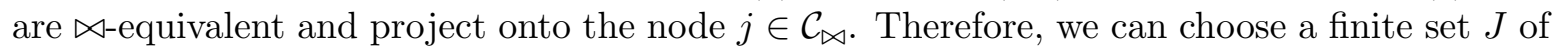
$j$ such that

$$
I(c)=\bigcup_{j \in J} \Omega_{c}(j)
$$

Since input sets lift, the existence of $\beta_{2}$ implies that

$$
I\left(c^{\prime}\right)=\bigcup_{j \in J} \Omega_{c^{\prime}}\left(\beta_{2}(j)\right) .
$$

We construct the permutation $\beta_{1}$ by finding bijections

$$
\left.\beta_{1}\right|_{\Omega_{c}(j)}: \Omega_{c}(j) \rightarrow \Omega_{c^{\prime}}\left(\beta_{2}(j)\right)
$$

for all $j \in J$ and letting $\beta_{1}$ be their union. The existence of $\beta_{2} \in B\left(d, d^{\prime}\right)$ implies that $(j, d) \sim_{E_{\bowtie}}\left(\beta_{2}(j), d^{\prime}\right)$. Recall from (7.4) that the definition of $\sim_{E_{\bowtie}}$ implies that there exists $\gamma \in B\left(c, c^{\prime}\right)$ such that $\gamma\left(\Omega_{c}(j)\right)=\Omega_{c^{\prime}}\left(\beta_{2}(j)\right)$. Thus $\beta_{2}(\bar{i})=\overline{\gamma(i)}$ for all $i \in \Omega_{c}(j)$. Now set $\left.\beta_{1}\right|_{\Omega_{c}(j)}=\gamma$.

\section{Nonuniqueness and universality.}

Theorem 8.4. Assume that $\bowtie$ is a balanced equivalence relation on $\mathcal{C}$, and let $G_{\bowtie}$ be the associated natural quotient network with quotient map $\phi$. Then the pair $\left(G_{\bowtie}, \phi\right)$ is universal. That is, if $G^{\prime}$ is a coupled cell network with a quotient map $\phi^{\prime}$ with $\bowtie_{\phi^{\prime}}=\bowtie$, then there is a quotient map $\xi: G_{\bowtie} \rightarrow G^{\prime}$ such that $\phi^{\prime}(c)=\xi(\phi(c))$ for all $c \in \mathcal{C}$.

In this situation we say that $\left(G^{\prime}, \phi^{\prime}\right)$ factors through $\left(G_{\bowtie}, \phi\right)$. Note that with the definition of $\phi$ given in Theorem 8.3,c®d if and only if $c \bowtie_{\phi} d$. 
First, we give an example to show that quotient networks need not be unique. Then we prove Theorem 8.4, which shows that the natural quotient is universal. That is, all other quotient networks are quotients of $G_{\bowtie}$ of a rather trivial kind: distinct cells remain distinct.

Example 8.5. Figure 21 shows three coupled cell networks. The network $G$ is the sevencell chain of Figure 6 . The network $G_{\bowtie}$ is the three-cell ring of Figure 7 in which all three arrows are equivalent. The network $G^{\prime}$ is another three-cell ring, in which the arrows are not equivalent. It is easy to see that there exist three quotient maps $\phi: G \rightarrow G_{\bowtie}, \phi^{\prime}: G \rightarrow G^{\prime}$, and $\xi: G_{\bowtie} \rightarrow G^{\prime}$, shown by the coloring of the figure. Moreover,

$$
\phi^{\prime}(c)=\xi(\phi(c)) \quad \forall c \in \mathcal{C} .
$$

Clearly $\phi$ and $\phi^{\prime}$ induce the same equivalence relation on $G$; that is, $\bowtie_{\phi}=\bowtie_{\phi^{\prime}}$. However, $G_{\bowtie}$ and $G^{\prime}$ are not isomorphic.

In fact, there are three other quotient networks with the same equivalence relation. Namely, form a three-cell ring and define two arrows to be equivalent but the third to be different. These three networks can be inserted between $G_{\bowtie}$ and $G^{\prime}$.

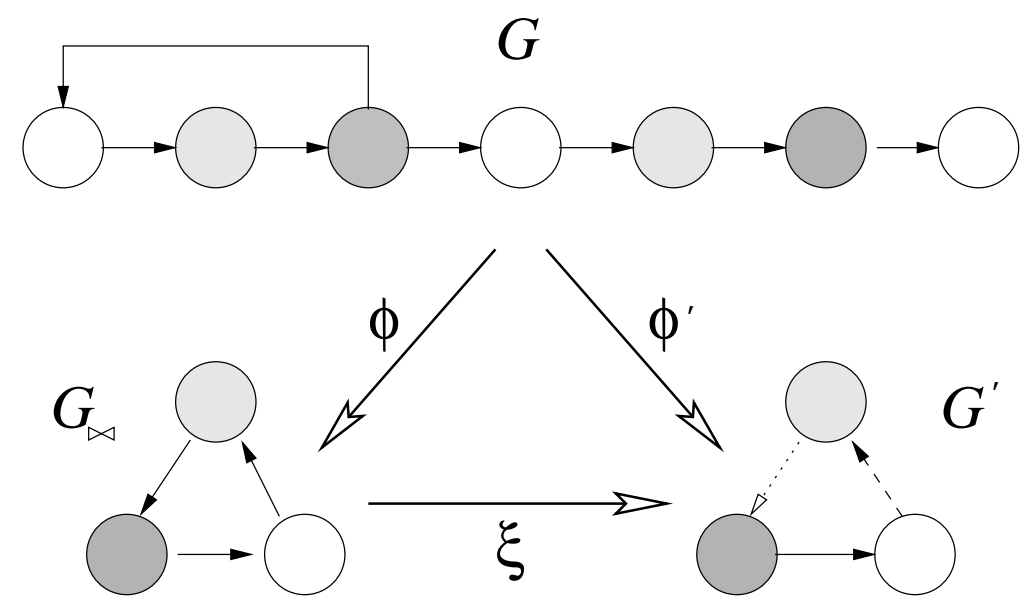

Figure 21. Two distinct quotients with the same equivalence relation.

The essential point now is that Example 8.5 exhibits the only way in which uniqueness fails. The natural quotient $G_{\bowtie}$ defined above is the one in which as many arrows as possible are edge-equivalent. All other quotients are obtained from the natural one by employing the same cells and refining $\sim_{E}$.

It will be helpful to introduce the following concept.

Definition 8.6. Let $\gamma: J \rightarrow K$ be a bijection between subsets $J, K \subset \mathcal{C}$, and let $\bowtie$ be an equivalence relation on $\mathcal{C}$. Say that $\gamma$ is $\bowtie$-compatible if for all $j_{1}, j_{2} \in J$

$$
j_{1} \bowtie j_{2} \Leftrightarrow \gamma\left(j_{1}\right) \bowtie \gamma\left(j_{2}\right) .
$$

Essentially, the point here is that $\gamma$ permutes $\bowtie$-equivalence classes. Note that in the definition of "balanced" we have the stronger condition $i \bowtie \gamma(i)$, in which $\gamma$ fixes $\bowtie$-equivalence classes. 
Such maps arise for the following reason. Suppose that $\phi: G \rightarrow G^{\prime}$ is any quotient map of coupled cell networks, and let $\beta \in \mathcal{B}_{G^{\prime}}$ be an input isomorphism. The definition of "quotient" requires there to exist a lift $\tilde{\beta} \in \mathcal{B}_{G}$. The definition of "lift" clearly implies that

$$
\tilde{\beta} \text { is } \bowtie_{\phi} \text {-compatible }
$$

with $J=I(c), K=I(d)$, whence $\tilde{\beta} \in B(c, d)$ (if $\beta \in B(\phi(c), \phi(d)) \subseteq \mathcal{B}_{G^{\prime}}$ ).

Proof of Theorem 8.4. Suppose that $G$ is a coupled cell network and $\bowtie$ is a balanced equivalence relation on $\mathcal{C}$. Let $\left(G_{\bowtie,} \phi\right)$ be the natural quotient by $\bowtie$ so that $\bowtie_{\phi}=\bowtie$. Let $\left(G^{\prime}, \phi^{\prime}\right)$ be any quotient network with $\bowtie_{\phi^{\prime}}=\bowtie$. We claim that $\phi^{\prime}$ factors through $\phi$.

Define $\xi: G_{\bowtie} \rightarrow G^{\prime}$ as follows. Let $\bar{c} \in \mathcal{C}_{\bowtie}$ be a cell of $G_{\bowtie \text {. Define }}$

$$
\xi(\bar{c})=\phi^{\prime}(c) .
$$

The map $\xi$ is well defined because $\bowtie_{\phi^{\prime}}=\bowtie=\bowtie_{\phi}$. It is a bijection $\xi: \mathcal{C}_{\bowtie} \rightarrow \mathcal{C}^{\prime}$.

We claim that $\xi$ is a quotient map. The defining properties are obvious, except for the condition that input isomorphisms lift from $G^{\prime}$ to $G_{\bowtie}$. Suppose that $\beta \in \mathcal{B}_{G^{\prime}}$. Then $\beta$ lifts from $G^{\prime}$ to $G$, yielding an input isomorphism $\tilde{\beta} \in \mathcal{B}_{G}$. By (8.6), $\tilde{\beta}$ is $\bowtie$-compatible. Therefore, it induces a bijection $\gamma$ on $\mathcal{C}_{\bowtie}$ defined by

$$
\gamma(\bar{c})=\overline{\tilde{\beta}(c)}
$$

The definition of edge-equivalence in the construction of $G_{\bowtie}$ implies that $\gamma$ is an input isomorphism in $G_{\bowtie}$. Therefore, every $\beta \in \mathcal{B}_{G^{\prime}}$ lifts to some $\gamma \in \mathcal{B}_{G_{\bowtie}}$.

Several other properties follow directly from this proof. To state them, we need the following definition:

Definition 8.7. Let $\xi: G \rightarrow G^{\prime}$ be bijective on cells. Then $G^{\prime}$ is an edge-refinement of $G$ if

$$
\xi(i, c) \sim_{E^{\prime}} \xi(j, d) \quad \Rightarrow \quad(i, c) \sim_{E}(j, d) .
$$

Corollary 8.8.

(a) Every quotient network corresponding to a given balanced equivalence relation $\bowtie$ is an edge-refinement of the natural quotient $G_{\bowtie}$.

(b) Conversely, every edge-refinement of $G_{\bowtie}$ is a quotient network corresponding to $\bowtie$.

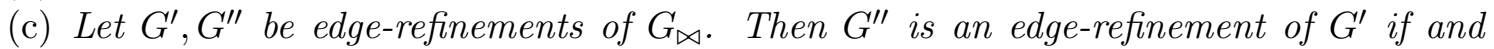
only if $\mathcal{B}_{G^{\prime}} \supseteq \mathcal{B}_{G^{\prime \prime}}$.

(d) The condition $\mathcal{B}_{G^{\prime}} \supseteq \mathcal{B}_{G^{\prime \prime}}$ is equivalent to $\mathcal{F}_{G^{\prime}}^{P} \subseteq \mathcal{F}_{G^{\prime \prime}}^{P}$ for any choice of phase space $P$ on cells, where cells in $G^{\prime}, G^{\prime \prime}$ are identified if they correspond to the same $\bowtie$-class of cells in $G$.

The above corollary establishes that the phenomena described in Example 8.5 are typical of the general case.

9. Induced vector fields are admissible. Now we come to the second main theorem of this paper. We show that any quotient map $\phi: G_{1} \rightarrow G_{2}$ converts $G_{1}$-admissible vector fields into $G_{2}$-admissible vector fields in a natural way.

The basic idea is the following. Let $\Delta_{\phi}$ denote the polydiagonal subspace corresponding to the equivalence relation $\bowtie_{\phi}$ (previously denoted $\Delta_{\bowtie_{\phi}}$ ). We claim that the space of 
$G_{1}$-admissible vector fields restricted to $\Delta_{\phi}$ can be naturally identified with a subspace of the space of $G_{2}$-admissible vector fields. The main consequence of this observation is that interesting dynamics (rotating waves, symmetric chaos) in this subspace for the cell system $G_{2}$ corresponds to the same dynamics in the cell system $G_{1}$, in which $\bowtie$-equivalent cells are synchronous.

We first choose cell phase spaces $P_{c}$ for $c \in \mathcal{C}_{1}$. Then $\phi(c) \in \mathcal{C}_{2}$, and we let the corresponding cell phase space be $\bar{P}_{\phi(c)}=P_{c}$. The space $\bar{P}_{\phi(c)}$ is well defined since quotient maps preserve the relation $\sim_{c}$.

Choose a set of representatives $\mathcal{R}$ for the map $\phi$. That is, $\mathcal{R} \subseteq \mathcal{C}_{1}$ and for each $d \in \mathcal{C}_{2}$ there exists a unique $c \in \mathcal{R}$ such that $\phi(c)=d$. Thus the set of all $\phi(c)$ runs through the elements of $\mathcal{C}_{2}$ without duplication when $c$ runs through $\mathcal{R}$. Then define

$$
\bar{P}=\prod_{c \in \mathcal{R}} \bar{P}_{\phi(c)}=\prod_{c \in \mathcal{R}} P_{c}
$$

If $x=\left(x_{c}\right)_{c \in \mathcal{C}_{1}}$ defines coordinates on $P$, we can consider $y=\left(y_{\phi(c)}\right)_{\phi(c) \in \mathcal{C}_{2}}$ as defining coordinates on $\bar{P}$. Moreover, for each $c \in \mathcal{C}_{1}$ there exists a unique $r \in \mathcal{R}$ such that $\phi(c)=\phi(r)$, and then $y_{\phi(c)}$ is identified with $y_{\phi(r)}$.

In section 8 we introduced the notion of a quotient map between coupled cell networks. The key property that we wish to ensure is that a quotient map $\phi: G_{1} \rightarrow G_{2}$ induces a natural mapping $\hat{\phi}: \mathcal{F}_{G_{1}}^{P} \rightarrow \mathcal{F}_{G_{2}}^{\bar{P}}$, where $\bar{P}$ is obtained by identifying the relevant factors of $P$.

Quotients preserve admissibility. We now establish an important property of quotient maps: they induce admissible vector fields.

Suppose that $\phi: G_{1} \rightarrow G_{2}$ is a quotient map. There is an injective map $\alpha: \bar{P} \rightarrow P$ defined by

$$
\alpha(y)_{c}=y_{\phi(c)} \quad \forall c \in \mathcal{C}_{1}, y \in \bar{P} .
$$

Note that $\Delta_{\phi}=\alpha(\bar{P})$, so $\alpha: \bar{P} \rightarrow \Delta_{\phi}$ is a bijection. Replacing $y$ by $\alpha^{-1} x$, for $x \in \Delta_{\phi},(9.1)$ becomes

$$
\left(\alpha^{-1} x\right)_{\phi(c)}=x_{c} \quad \forall c \in \mathcal{C}_{1} .
$$

Definition 9.1. Since $f \in \mathcal{F}_{G_{1}}^{P}$ leaves $\Delta_{\phi}$ invariant, we can define a vector field $\bar{f}$ on $\bar{P}$, the induced vector field, by restricting $f$ to $\Delta_{\phi}$ and projecting the result onto $\bar{P}$ by $\alpha^{-1}$. More precisely,

$$
\bar{f}(y)=\alpha^{-1}(f(\alpha(y))) \quad \forall y \in \bar{P} .
$$

We will also denote $\bar{f}$ by $\hat{\phi}(f)$.

The main result of this section is Theorem 9.2 below. Several applications of this theorem can be found in [6].

Theorem 9.2. For any $f \in \mathcal{F}_{G_{1}}^{P}$, the induced vector field $\bar{f}$ lies in $\mathcal{F}_{G_{2}}^{\bar{P}}$.

Proof. Since $f \in \mathcal{F}_{G_{1}}^{P}$ is $G_{1}$-admissible, it satisfies the two conditions of Definition 4.1: the domain condition (4.3) and the equivariance condition (4.5). 
The domain condition states the following: For all $c \in \mathcal{C}_{1}$ there exists a function $\hat{f}_{c}$ : $P_{I(c)} \rightarrow P_{c}$ such that

$$
f_{c}(x)=\hat{f}_{c}\left(x_{I(c)}\right)
$$

Equivalently, $f_{c}$ depends only on variables from cells in $I(c)$.

$\mathcal{B}_{G_{1}}$-equivariance states the following: For all $c, c^{\prime} \in \mathcal{C}_{1}$ and for all $\gamma \in B\left(c, c^{\prime}\right)$ we have

$$
\hat{f}_{c^{\prime}}(x)=\hat{f}_{c}\left(\gamma^{*}(x)\right) \quad \forall x \in P_{I\left(c^{\prime}\right)},
$$

where

$$
\left(\gamma^{*}(x)\right)_{i}=x_{\gamma(i)} \quad \forall i \in I(c)
$$

and is undefined elsewhere.

We must verify Definition 4.1. That is, we must show the following:

(a) The vector field $\bar{f}$ satisfies the domain condition for $G_{2}$.

(b) The vector field $\bar{f}$ satisfies the equivariance condition for $G_{2}$.

To prove (a), let $d \in \mathcal{C}_{2}$, and suppose that $\phi(c)=d$. We must show that $\bar{f}_{d}(y)$ depends only on $y_{I(d)}$ for $y \in \bar{P}$. We have

$$
\begin{array}{rlrl}
\bar{f}_{d}(y) & =\bar{f}_{\phi(c)}(y) & \\
& =(\bar{f}(y))_{\phi(c)} & \\
& =\left(\alpha^{-1}(f(\alpha(y)))\right)_{\phi(c)} & & \text { by }(9.3) \\
& =(f(\alpha(y)))_{c} & & \text { by }(9.2) \\
& =f_{c}(\alpha(y)) & & \text { by definition. }
\end{array}
$$

By (9.4), the value of $f_{c}(\alpha(y))$ depends only on $(\alpha(y))_{I(c)}$. However, by (9.1),

$$
(\alpha(y))_{I(c)}=y_{\phi(I(c))}=y_{I(d)}
$$

since $\phi(I(c))=I(d)$ by (8.3). This proves (a).

We interrupt this proof to verify a lemma.

Lemma 9.3. Let $d, d^{\prime} \in \mathcal{C}_{2}$ and let $\beta \in B\left(d, d^{\prime}\right)$. Choose $c, c^{\prime} \in \mathcal{C}_{1}$ such that $\phi(c)=d, \phi\left(c^{\prime}\right)=$ $d^{\prime}$. Suppose that $\beta$ lifts to $\tilde{\beta} \in B\left(c, c^{\prime}\right)$. Then for all $y \in \bar{P}$ we have

$$
\tilde{\beta}^{*}(\alpha(y))=\alpha\left(\beta^{*}(y)\right) .
$$

Proof. For all $i \in I(c)$ we have

$$
\begin{array}{rlrl}
\left(\tilde{\beta}^{*}(\alpha(y))\right)_{i} & =(\alpha(y))_{\tilde{\beta}(i)} & & \text { by }(9.6) \\
& =y_{\phi(\tilde{\beta}(i))} & & \text { by }(9.1) \\
& =y_{\beta(\phi(i))} & \text { by }(8.1) \\
& =\left(\beta^{*}(y)\right)_{\phi(i)} & \text { by }(9.6) \\
& =\left(\alpha\left(\beta^{*}(y)\right)\right)_{i} & & \text { by }(9.1),
\end{array}
$$

which proves (9.7). 
Now we return to the proof of Theorem 9.2. To prove (b) we must show that for all $d, d^{\prime} \in \mathcal{C}_{2}$ and $\beta \in B\left(d, d^{\prime}\right)$,

$$
\bar{f}_{d^{\prime}}(y)=\bar{f}_{d}\left(\beta^{*}(y)\right) \quad \forall y \in P_{I\left(d^{\prime}\right)},
$$

where by definition

$$
\left(\beta^{*}(y)\right)_{i}=y_{\beta(i)}
$$

Choose $c, c^{\prime} \in \mathcal{C}_{1}$ such that $\phi(c)=d, \phi\left(c^{\prime}\right)=d^{\prime}$. Use Definition 8.1 (c) to lift $\beta: I(d) \rightarrow$ $I\left(d^{\prime}\right)$ to $\tilde{\beta}: I(c) \rightarrow I\left(c^{\prime}\right)$. That is, by (8.1),

$$
\beta(\phi(i))=\phi(\tilde{\beta}(i)) \quad \forall i \in I(c) .
$$

We know that

$$
f_{c^{\prime}}(x)=f_{c}\left(\tilde{\beta}^{*}(x)\right) \quad \forall x \in P .
$$

Therefore, setting $x=\alpha(y)$, we have

$$
f_{c^{\prime}}(\alpha(y))=f_{c}\left(\tilde{\beta}^{*}(\alpha(y))\right) \quad \forall y \in \bar{P} .
$$

By Lemma 9.3,

$$
f_{c^{\prime}}(\alpha(y))=f_{c}\left(\alpha\left(\beta^{*}(y)\right)\right)
$$

Now, by definition (9.3), $\bar{f}(y)=\alpha^{-1}(f(\alpha(y)))$. Therefore,

$$
\begin{array}{rlr}
\bar{f}_{d^{\prime}}(y) & =\left(\alpha^{-1}(f(\alpha(y)))\right)_{d^{\prime}} & \\
& =\left(\alpha^{-1}(f(\alpha(y)))\right)_{\phi\left(c^{\prime}\right)} & \\
& =(f(\alpha(y)))_{c^{\prime}} & \text { by }(9.2) \\
& =f_{c^{\prime}}(\alpha(y)) &
\end{array}
$$

Similarly,

$$
\begin{aligned}
& \bar{f}_{d}\left(\beta^{*}(y)\right)=\left(\bar{f}\left(\beta^{*}(y)\right)\right)_{d} \\
& =\left(\alpha^{-1}\left(f\left(\alpha\left(\beta^{*}(y)\right)\right)\right)\right)_{d} \\
& =\left(f\left(\alpha\left(\beta^{*}(y)\right)\right)\right)_{c} \quad \text { by }(9.2) \\
& =f_{c}\left(\alpha\left(\beta^{*}(y)\right)\right) \\
& =f_{c}\left(\tilde{\beta}^{*}(\alpha(y))\right) \quad \text { by }(9.7) \text {, }
\end{aligned}
$$

and the result follows from (9.9).

Theorem 9.2 is valid for all of the quotients in Example 8.5. However, it is clear that $\mathcal{B}_{G^{\prime}}$ is a proper subset of $\mathcal{B}_{G_{\bowtie}}$. Therefore, $\operatorname{im}(\hat{\phi})=\operatorname{im}\left(\hat{\phi}^{\prime}\right) \subseteq \mathcal{F}_{G_{\bowtie}} \subset \mathcal{F}_{G^{\prime}}$, so we gain more information about induced vector fields $\bar{f}$ and their lifts $f$ if we work with $\left(G_{\bowtie}, \phi\right)$ rather than $\left(G^{\prime}, \phi^{\prime}\right)$.

Note that Example 7.5 is the natural quotient, so $\hat{\phi}$ need not be surjective when $\phi$ is natural. It is never surjective when $\phi$ is not the natural quotient map. 
10. Final comments. The formalism of symmetry groupoids proposed in this paper can be set up for many analogous systems that possess a network structure. Here, we have associated to each cell (node of the network) a continuous-time dynamical system defined on a manifold, and to each directed edge a coupling between such systems. We briefly consider variations on this theme.

Extra constraints can be imposed, an important one being to make the system Hamiltonian; see [8]. An analogous formalism can be introduced for discrete-time dynamics (coupled map lattices and generalizations to networks), or discrete-time discrete-space dynamics (cellular automata), and groupoid-equivariance implies constraints on the dynamics (in particular, on patterns of synchrony). If cells represent states of a stochastic process and edges represent transitions, then the network corresponds to a Markov chain, and now the symmetry groupoid implies constraints on the stationary probability density function. We can also extend the groupoid formalism to stochastic differential equations and delay-differential equations.

The theory developed here is a preliminary step toward a formal understanding of patternformation in general, not necessarily symmetric, coupled cell networks. Its main focus is robust synchrony. Many other questions about the dynamics of coupled cell networks can be tackled within the groupoid framework; indeed, work is in progress on several of these. In all cases, the central role of the symmetry groupoid as a formal algebraic structure that captures the constraints imposed by the network topology is paramount.

Acknowledgments. We thank Ronnie Brown for advice about groupoids and suggestions for appropriate literature, Ana Dias for commenting on an early version of the paper, and Andrew Török for identifying the $\mathbf{D}_{3}$ symmetry in the quotient in Example 7.1. The work of Ian Stewart was carried out in part during visits to the Banach Center, Warsaw and the Mathematics Institute of the Jagiellonian University, Kraków, organized, respectively, by Staszek Janeczko and Krzysztof Cesielski; he thanks them and their institutions for hospitality and financial support. Finally, we thank all three referees of this paper for their careful scrutiny, which led to substantial improvements.

\section{REFERENCES}

[1] H. Brandt, Über eine Verallgemeinerung des Gruppenbegriffes, Math. Ann., 96 (1927), pp. 360-366.

[2] S. Bocchletti, L. M. Pecora, AND A. Pelaez, Unifying framework for synchronization of coupled dynamical systems, Phys. Rev. E (3), 63 (2001), 066219.

[3] R. Brown, From groups to groupoids: A brief survey, Bull. London Math. Soc., 19 (1987), pp. $113-134$.

[4] P. L. Buono And M. Golubitsky, Models of central pattern generators for quadruped locomotion I. Primary gaits, J. Math. Biol., 42 (2001), pp. 291-326.

[5] A. Dias AND I. Stewart, Symmetry groupoids and admissible vector fields for coupled cell networks, submitted.

[6] M. Golubitsky, M. Nicol, And I. Stewart, Some curious phenomena in coupled cell networks, submitted.

[7] M. Golubitsky And I. Stewart, The Symmetry Perspective: From Equilibrium to Chaos in Phase Space and Physical Space, Progr. Math. 200, Birkhäuser Verlag, Basel, 2002.

[8] M. Golubitsky And I. Stewart, Patterns of oscillation in coupled cell systems, in Geometry, Dynamics, and Mechanics: 60th Birthday Volume for J. E. Marsden, P. Holmes, P. Newton, and A. Weinstein, eds., Springer-Verlag, New York, 2002, pp. 243-286. 
[9] M. Golubitsky, I. N. Stewart, and D. G. Schaeffer, Singularities and Groups in Bifurcation Theory: Vol. 2., Appl. Math. Sci. 69, Springer-Verlag, New York, 1988.

[10] P. J. Higgins, Notes on Categories and Groupoids, Van Nostrand Reinhold Mathematical Studies 32, Van Nostrand Reinhold, London, 1971.

[11] S. MacLane, Categories for the Working Mathematician, Springer-Verlag, New York, 1971.

[12] L. M. Pecora And T. L. Carroll, Synchronization in chaotic systems, Phys. Rev. Lett., 64 (1990), pp. $821-824$.

[13] W. T. Tutre, Graph Theory, Encyclopedia Math. Appl. 21, G.-C. Rota, ed., Addison-Wesley, Reading, MA, 1984.

[14] X. F. WAng, Complex networks: Topology, dynamics and synchronization, Internat. J. Bifur. Chaos Appl. Sci. Engrg., 12 (2002) pp. 885-916.

[15] A. Weinstein, Groupoids: Unifying internal and external symmetry, Notices Amer. Math. Soc., 43 (1996), pp. 744-752.

[16] R. J. WiLson, Introduction to Graph Theory, 3rd ed., Longman, Harlow, UK, 1985. 